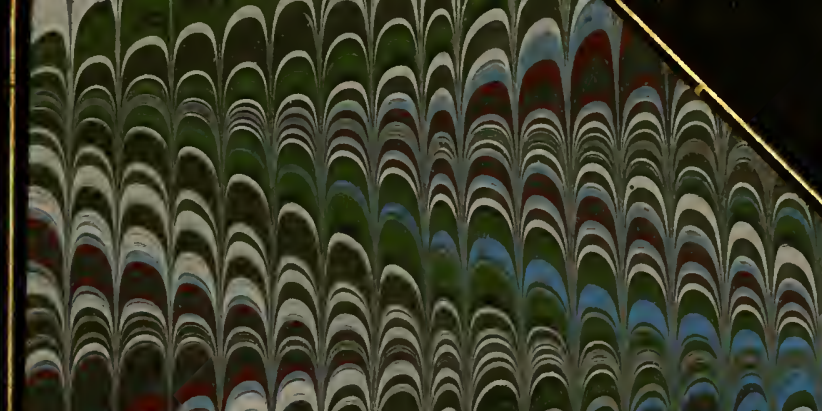

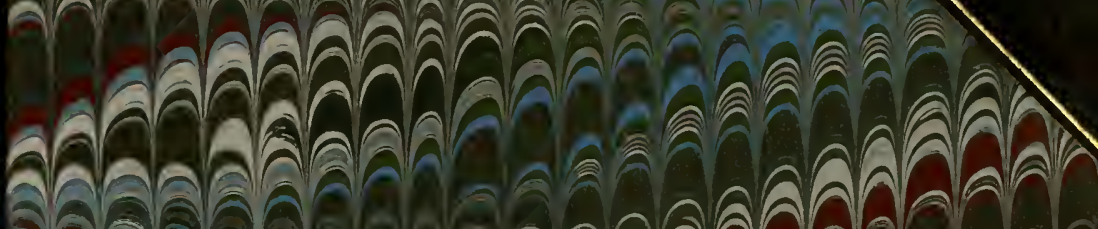
ลิกลกลล

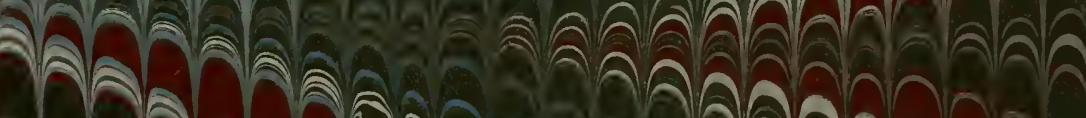

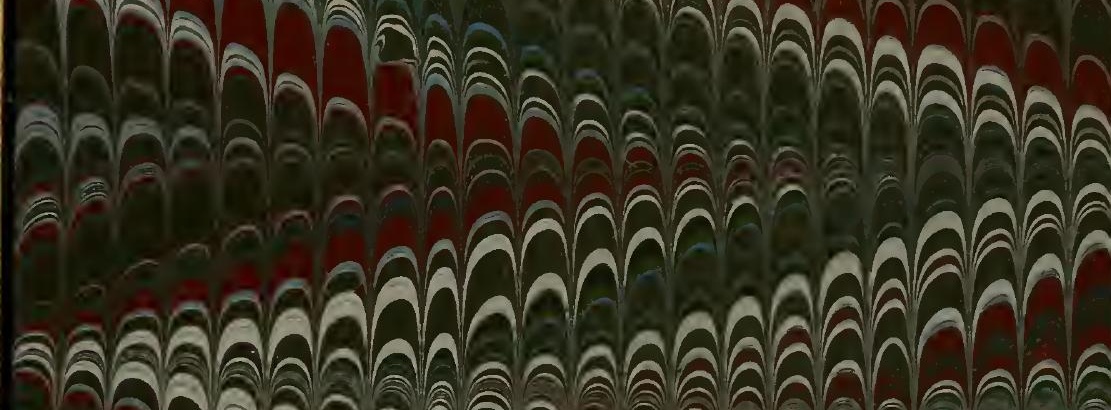
คคค ค

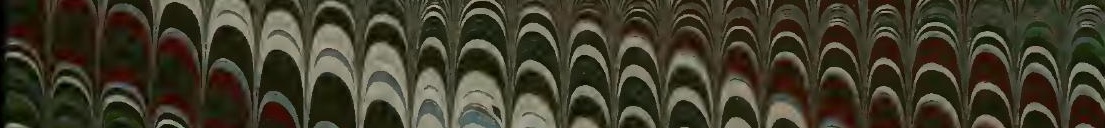

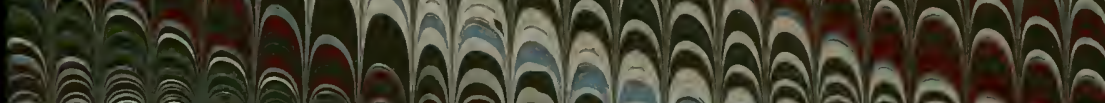

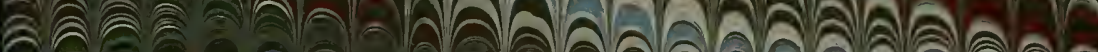

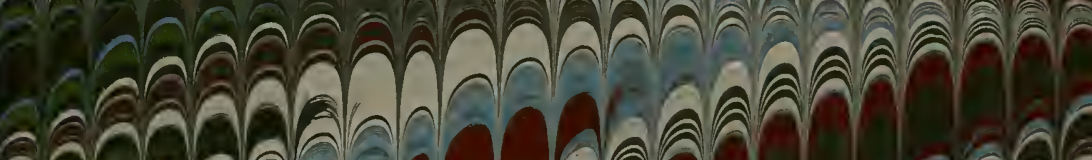

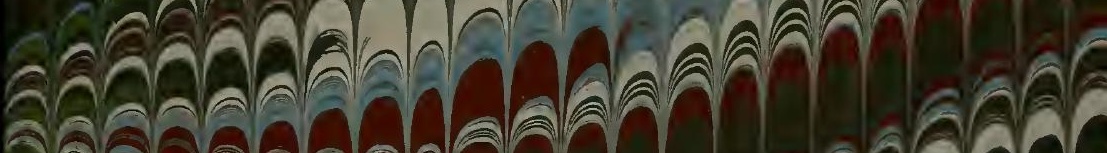

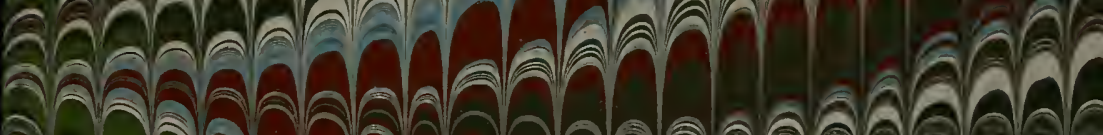

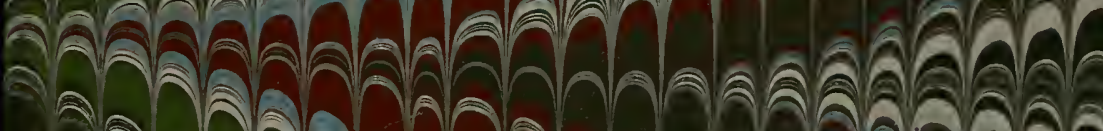

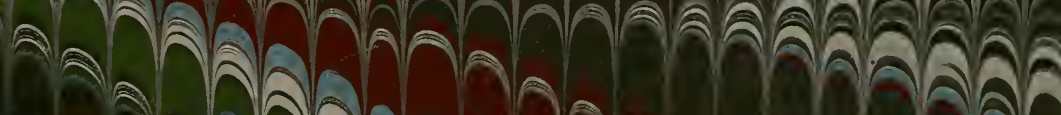
ลล ลลลลลส

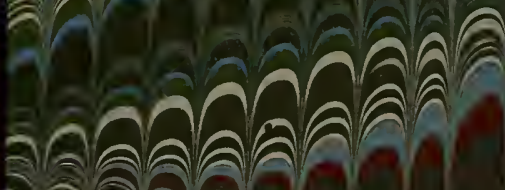

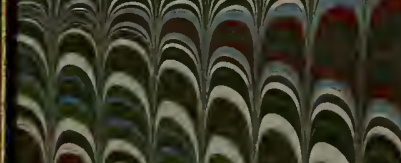

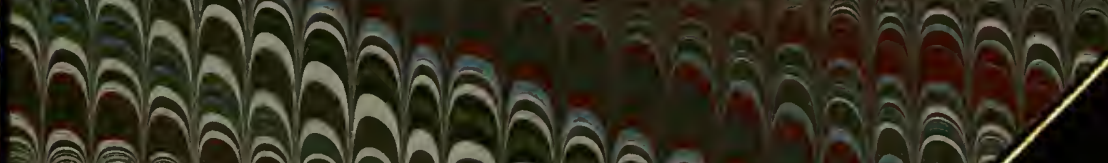

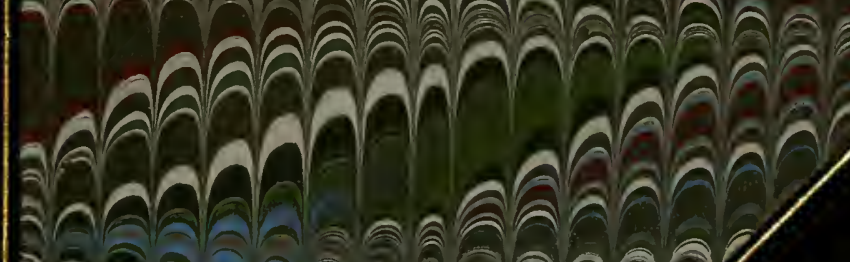

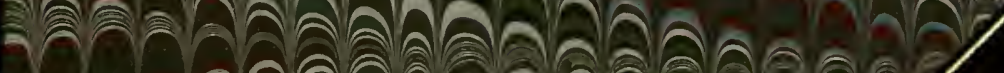

กลก 







\title{
$\begin{array}{llllllll}\mathrm{XIX} & \text { T } & \mathbf{A} & \mathbf{B} & \mathbf{U} & \mathbf{L} & \mathbf{E}\end{array}$
}

\section{ANATOMIIM ENTOZOORUM}

\author{
ILLUSTRA N TES,
}

CONGESTAE, NEC NON EXPLICATIONE PRAEditaE

A B

\section{EDUARDO SCHMALZ,}

DOCTORE PHILOSOPHIAE, MIEDICINAE ET CHIRURGIA,

IIEDICO PRACTICO D ESDENSI, SOCIETATIS MEDICONUAI GALLICO-LUGDUNENTSIS IT IIBURNENSJS, ATIIEADEI TREVISANT, SOCIETATIS PHYSIOGPAPHICAE LUNDENSIS, REGIAE BOTANICAE RATISBONENSIS, NATURAE SCRUTATORUM LIPSIENSIS, NATURAE SCTU-

TATORUM ET MEDICORUM DRESIENSIS SODALI.

DRESDAE IT LIPSIAE,

$\begin{array}{llllllllllllllll}\text { I N } & \text { L I } & B & R & A & R & \text { I } & A & A & R & N & O & L & D & I\end{array}$

1831. 


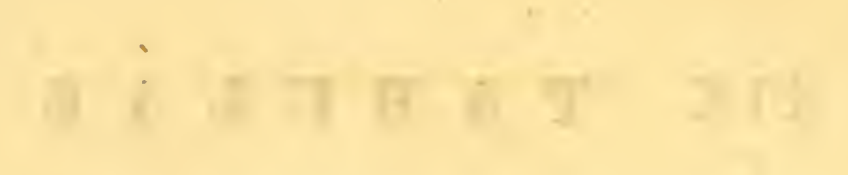

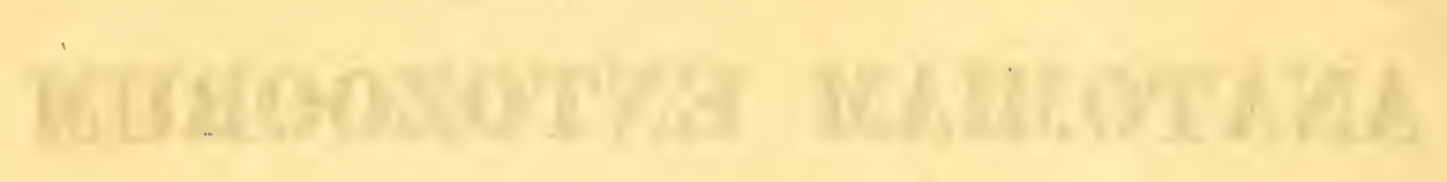

: Mownengtyans

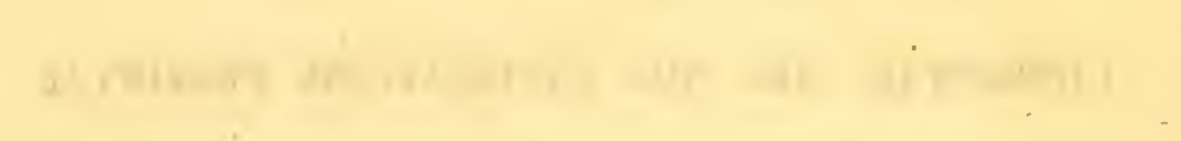

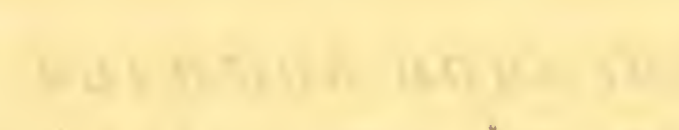




\section{LECTORI BENEVOLO.}

Recentiori tempore plurima et graviora de Anatomia et Physiologia Entozoorum observatil extant. Jam vero cum, quae habemus, plurimis sint dispersa libris et ephemeridibus, ante hos ultimos annos dispersa colligere et in unum idemque opus comprehendere hatd alienum judicavi. Quod quidem eo magis utile mihi visum est atque salubre, quo certius apud omnes constat, tironibus, physiologiae et historiae naturaii operam navantibus, neutiquam datam esse copiam suppellectilis literariae acquirendae, nec otium concessum e fontibus ipsis hauriendi. Quae cum ita sint, opusculum hujusmodi indolis componere animus fuit.

Quam rem aggressus, campum hunc literarium ambitus esse haud exigui, nec brevi tempore apte peragrari posse, cito intellexi. Opportunitate autem iter majus faciendi panlo post mihi dấa sâuiorum meorum horas ex toto quasi absorptas vidi. Igitur ad reditum usque editionem eorum, quae jim collecta erant, distuli, parte minore tintummodo excepta, quam de Fintozoomum systemate nervoso, dissertationis inauguralis nomine, in publicum producere ausus sum. Deo 0 . Mil. farente ab hoc itinerc in patriam redux, quo minus ad inchoatum hoc opus perficiendtum me accingam, plurimis impeditus sum occupationibus, ut nunc saltem nihil in me recipere possin. 
Jam rero solam collectionem tabularum, quae praecipua cujusvis classis rermium intestinalium organa ante oculos ponant, tironibus haud inutile fore ratus, has tabulas, una cum earum explicatione, lectoribus benevolis tradere constitui.

Qua quidem occasione mihi oblata non possum non publice profiteri, quali et quanta gratia me obstrictum sentiam viribus excellentissimis et illustrissimis: Dr. Chr. Lutl. Nitzsch, Prof. Zool. P. O. in Univ. Halensi, etc, Dr. Sam. Thom. de Soemmerring, Reg. Barar. a consil. intim, etc. (eheu! interim mortuo), et Dr. Fischer, Imp. Austr. a consil,, Prof. in Acad. med.-chir. Joseph. Viennensi etc., qui quidem nonnullas icones originales his tabulis excudendi veniam summa mihi dederunt benerolentia.

Dresdae, Calendis Januariis 1831. 


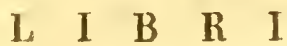

EX QUIBUS TABULAE DESUMTAE SUNT.

1. Lud. Heinr. Bojanus, Enthelminthica. Isis 1821. 2. Heft, p. $162-154$. mit 2 Kupfertafeln.

2. Dr. Bremser, über lebende Würmer im lebenden Menschen. Mit 4 Kupfertafeln. Wien, 1819. 4 .

3. Joan. Godofr. Bremser, Jcones helminthum systema Rudolphii entozoologicum illustrantes. XX tabb. Vienn, 1824. Fol.

4. Jul. Cloquet, Anatomie des rers intestinaux: Ascaride lombricoïde et Echinorhynque géant. Arec huit planches. Paris, 1824. 4.

5. Fr. Clr. Ilenr. Creplin, Observationes de Eutozois. Pars I, cum tab. aen. Gryphiswaldiae, 1825. S.

6. Joh. Aug. Ehır. Goeze, Vers. einer Naturgeschichte der Eingeweidewürmer. Mit 44 Kupfertafelı. Blankenburg, 17S2. S.

7. Louis Jurine, note sur la Douve à loug cou (Fasciola lucii) in: Annales des sciences natur. par MIY. Audouin, Broguiart et Dumas. Tom. II., Paris, 1824. p. 493. sq., arec une planche.

S. Fr. Sigm. Leuckart, Zoologische Bruchstücke. Mit 2 Kupfertafeln. Helmstädt, 1820. 4.

9. Eduardus Mehlis, Observationes anatom. de Distomate hepatico et lanceolato. Götting. 1825. Fol, cum tab, aenca.

10. C. G. Ni: ssch, in Ersch u. Gruber Encjclopaedie. Artikel: Acanthocephala, Amphistoma, Ascaris etc. 
11. J. Fr. de Olfers, Comment, de regetatiris et animatis corporibus in corporibus animatis reperiundis. P. 1. Berol. 1526. 8.

12. Otto, Ueber die Nerren der Eingewciderürner, im Berliner Magazin, 1S14. p. 17S. sq. Mit 2 Kupfertafelı.

13. C. A. Rudolphi, Entozoorum s. rermium intestimalium historia naturalis. III roll. cum XII tabb. aen. Arstelod. $1808-1810$. S.

14. C. A. Rudolphi, Entozoorum synopsis. Cum III tabb, aen. Berol. 1S19. 8.

15. Joan. Grg. Steinbuch, Comment. de taenia hydatigena anomala, adnexis cogitatis quibusdam de rermium visceralium physiologia. Cum tab, aen. Erlangae, 1802. S.

16. Aug. Henr. Westrumb, de helminthibus acanthocephalis. Cum III tab. acn. Hanoverae, 1821. Fol.

17. Aug. Henr. Westrumb, Beitrag zur Anatomie des Strongylus armatus, in: Isis 1S22. 6. Heft, p. 685. sq. Mit 1 Kupfertafel.

18. Joh. Grg. Heinr. Zeder, Erster Nachtrag zu J. A. E. Goese Naturgeschichte der Eüngeweidewürmer, Mit 6 Kupfertafeln. Leipzig, 1800. 


\section{T A B. I.

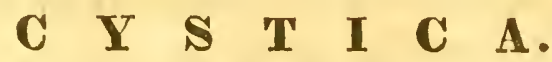

Fig. 1 -6. secundum Bremser Ueber lebende Würmer im lebenden Menschen (no. 2). - Fig. \%. 8. 10. secundum Bremser Icones helminthum (no. 3). - Fig. 9. et 11 - 13. secundum Rudolphi Entozoorum synopsis (no. 14).

- Fig. 14 - 1\% secundum Bojanus Enthelminthica (no. 1).

FIG. 1. 6. ECHINOCOCCUS HOMINIS. In sacco interne laeri, in hepate feminae obveniente, libere contentus.

Fig. 1. Hydatis parva, magnitudine naturali. Lamina vermis interior ab exteriore separata et contracta est.

Fig. 2. Hydatis major, magnitudine naturali. Ea, simul ac ex sacco suo exemta, recens delineata est.

Fig. 3. Eadem per noctem in aqua collocata. Lamina ejus interior, ut in fig. 1., ab exteriore separata est.

Fig. 4. IIydatis corpuscula majora, jam nudis oculis videnda, continens.

Fig. 5. Particula rermis, ex fig. 2. desumta, adacta. Quae nudis oculis in fig. 2. grana filla videbantur hic microscopii ope globulos parvos pellucidos esse conspicimus.

Fig. 6. Globulus singulus ex fig. 4. adauctus.

FIG. 7. ECIINOCOCCUS VETERINORUM. Ex hepate et pulmonibus Cameli Dromedarii.

Guttafluidi, in hydatide contenti, sub microscopio compositovisa. Animalcula s. Echinococcos ipsos, forma diversissimos, nec uon cchinos deciduos, magnitudine valde auctos sistit.

Fig. S-13. COENURUS CERebralis, Ex cerebro Ovis vertiginosae.

Fig. 8. Vesica vermiun integra, magnitudine imminuta.

Fig, 9. Vermis singulus, ex vesica sua perfecte exsertus, Magnitudine auctus. 
a. Caput, rostello uncinato, quatnorque osculis suctoriis instructum.

b. Corpus elongatum, depressiusculum, rugosum.

c. Vesicae conmunis pars, in quam rermis desinit.

Fig. 10. Vermes tres, in diverso exsertionis statu. Aucti.

a. a.a. Vermes.

b.b.b. Vesicae communis, in quam desinunt, pars.

Fig. 11. Caput a vertices. facic antica risum. Valde auctum.

a. a. a. a. Oscula ejusdem quatuor, inter quae

b. Corona uncinulorum duplex cum papilla media.

Fig. 12. I dem, a latere risum.

a. Rostelli apex protrusus, cum uncinulorum corona.

b. Oscula.

Fig. 13. I lem, a laterc visum, rostello ad partem retracto.

a. Rostellum, qua partem retractum, ideoque truncatum risum, uncinulis energentibus.

b. Oscula.

c. Pars corporis.

FIG. 14-17. CYSTICERCUS PILEATUS. Sub cute Simiae Inui, musculo bicipiti cruris adhaerens, inrentus.

Fig. 14. Capsulas. vesica externa vermem contincns. Magnitudine naturali. Ea simplex, nitide alba, et fibris circularibus distinctis pracdita est.

Fig. 15. Vermis e capsula desumtus, magnitudine naturali.

a. Pileus, in disci forma compressus, caput includens et collum corpusque condens, colore rubescenti - luteo.

b. Vesica caudalis, pyriformis, pellucida.

c. Vesiculae forma irregulari, pellucentes, sed non libere in resica caudali natantes.

Fig. 16. Idem, pileo reflexo, ita ut corpus in conspectum reniat.

a. Pileus.

b. Vesica caudalis.

d. Collum et corpus rermis, utrumque brevissimum, transrersim rugosum et strangulatum.

Fig. 17. Vermis pileo dissecto, multum adauctus.

a. Pileus dissectus.

b. Pars resicae caudalis.

d. Collum et corpus rermis.

c. Caput in pilei cavitate libere situm, subglobosum, nitide album, nec rostello uncinato, nec osculis suctoriis, nec bothriis instructum. 


\section{T A B. II.

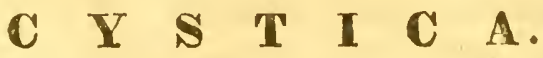

Fig. 1 - 4. secundum Bremser Lebende Würmer im lebenden Menschen (no. 2). - Fig. 5 - \% secundum Steinbach de taenia hydatigena (no. 15). Fig. 8-12. secundum Eremser Icones helminthum (no. 3).

FIG. 1-7, Crsticercus Cellulosae. Ex carne Suis Scrophae.

Fig. 1. Vermis vesica sua inclusus, magnitudine naturali:

Fig. 2. Vermis resica exemtus, magn. nat.

Fig. 3. Idem collo semi-eroluto.

Fig. 4. Idem collo et capite evolutis.

a. Caput tetragonum.

b. Collum brevissimum.

c. Corpus cylindricum rugosum.

d. Vesica caudalis.

Fig. 5. Idem, corpore perfecte eroluto, cum exigua parte resicae caudalis corpori adhaerente. Magnitudine auctus.

a. Rostellum,

b. Annulus raginalis, cui unci infixi sunt.

c. d. Corona uncorum.

c. Retinacula.

d. Apices eorum.

e. e. Ostia suctoria tria. Quartum in arerso capitis latere situm rideri non potest.

f. f. Canales sic dicti suctorii, quorum tres per tunicam transparent, antrorsum ad ostia suctoria vergentes.

g. Collum attenuatum, prorsus fere glabrum.

h. h. Corpus rugosum.

i. Finis corporis, cum resicula caudali in circulo connatus,

k. Vesiculae caudalis fragmentum, fini corporis adhaerens. 
Fig. 6. Caput ejusiem, a corpore separatum.

a. Rostellum.

b. Amulus raginalis.

c. Ostia raginarum, propter amissos uncos patula.

d. d. Ostia suctoria. Quartum hic per mediam aliquantulum transparet.

c.e.e. Canales suctorii, ab ostiis suis in collum euntes.

Fig. 7. Uncus singulus, e vagina sua remotus, a latere visus. Magnitudine multum adauctus.

a. Unci retinaculum.

b. Finis aliquantulum bullosus, anuulo raginali antea insertus.

c. Apex.

FIG. 8. et 9. CISTICERCUS FASCIOLARIS. Ex hepate Muris Musculi.

Fig. 8. Vermis integer, resica sua exemtus, magnitudine naturali.

a. Caput subtetragonum, collo carens.

b. Corpus depressum.

c. Vesica caudalis exigua subglobosa:

Fig. 9. Caput, cum parte corporis. Magnitudine aucta.

a. Rostellum unciratum.

b. Ostia suctoria.

c. Corporis pars.

FIG. 10. CYSTICERCUS TENUICOLLIS, E mesenterio Ovis Ammonis.

Maguitudo dimidia parte imminuta.

a. Caput tetragonum.

b. Colium breve, filiforme.

c. Corpus teretiusculum.

d. Vesica caudalis subglobosa, amplissima.

FIG. 11. et 12. CYSTICERCUS LONGICOLLIS. E caro thoracico Muris arvalis.

Fig. 11. Vermis e vesica sua exemtus, magnitudine adauctus.
a. Rostellum brevissimum, latum, uncinulorum corona duplici armatum.
b. Oscula suctoria.
c. Collum depressum, capite corporeque tenuius.
d. Corpus rugosum, flaccidum, depressum.
e. Vesica caudalis oralis.

Fig. 12. Idem, cum appendicibus resice caudalis.
a. Rostellum.
b. Oscula suctoria.
c. Collum.
d. Corpus.
e. Vesica caudalis.
f. Appendices ejusdem posticae duac. 


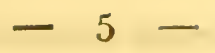

FIG. 13. et 14. ANTHOCEPHALUS MACROUliUs. E carnibus Bramae Raji.

Fig. 13. Vermis integer. Magnitudine naturali.

a. b. Caput.

a. Bothria, quorum quatuor distincta adsunt, et ex quibus prodeunt

b. Proboscides totidem longae.

c. Collum longum, capite tenuius.

d. Corpus breve, ovale, in quod caput ef collum retrahi possunt, terminatum c. c. Vesica caudali cylindrica, longissima.

Fig. 14. Caput cum parte corporis, auctum.

a.b. Caput.

a. Bothria suborata, concara.

b. Proboscides inde emissae, uncinis ternis spiraliter positis armatae.

c. Collum.

d. Corporis pars. 


\section{T A B. III. \\ $\begin{array}{lllllllllllllll}C & \mathbf{E} & \mathbf{S} & \mathbf{T} & \mathbf{O} & \mathbf{I} & \mathbf{D} & \mathbf{E} & \mathbf{A}\end{array}$}

Fig. 1 - 15. secundum icones originales, nondum incisas, quas summa benevolentia Vir Exc. atq. Exp. Dr. Chr. Ludlovic. Nitzsch, Prof. Zool. 1. O. apud Halenses, publicandas mihi commmicavit. - Fig. 16. secundum Zeder Ersier Nachtrag zu Göze Naturg. d. Eingew. (no. 18).

FIG. 1-6. TAENia Villosa. Ex Otidis Tardae intestinis.

Fig. 1. Articuli sex magnitudine aucti. Organa genitalia nondum evoluta.

Fig. 2. Iidem, genitalibus magis erolutis.

Fig. 3. Iidem, genitalibus perfecte evolutis.

Fig. 4. Genitalia, ex fig. 1. separatim delineata.

Fig. 5. Genitalia, É

Fig. 6. Gepitalia perfecta, ex fig. 3.

a. Testiculus.

x. Vas deferens.

b. Vagina penis.

c. Penis pars in corpore adluc contenta:

d. Vagina uteri.

e. Uterus.

h. i. Oraria.

f. Corporis finis.

g. Penis pars ex corpore erserta.

FIG. 7-11. 'TAENIA MIMANTOPODIS MELANOPTERI.

Fig. 7. Articuli nonnulli anteriores aucti. Genitalia obscura.

Fig. 8. Articuli medii.

Fig. 9. Articuli posteriores.

Fig. 10. Penis magis auctus.

a. Vagina penis.

b. Penis claratus, aculcis obsitus, 
Fig. 11. Idem, membrana ejus exterina soluta.

a. Vagina penis.

b. Penis, membrana exterua privalus.

c. Membrana catem aculeis obsita, a pene soluta, quac cjus ostium intrat et lobuli irregularis instar ci adlaeret.

FIG. $12-15$. 'TAENIA LARI RIDIBUNDI.

Fig. 12. Nonulli articuli aucti, genitalibus prominentibus.

Fig. 13. F'cuis sub microscopio risus.

a. Vagina penis.

b. Fijus basis.

c. Apex.

Fig. 14. Idem, cum canali interno obscure tantummodo apparente.
a. Tagina penis.
b. Ejus basis.
c. Canalis obscurus, qui per ejus longitudinem currens, eranescit.
d. Apex.

Fig. 15. Ora, sub microscopio risa.
a. Chorion.
b. Amnion tuberculosum.

Fig. 16. Articuli duo TAENAE SINUOSAE. Ex intestinis Anatis.
a. Oraria sacciformia, quae transcunt in
b.c. Lemniscos, qui ad basin articulorum prominent.
d.d. Anguli articulorum. 


\section{T A B. IV. \\ $\begin{array}{llllllllll}\mathbf{C} & \mathbf{E} & \mathbf{S} & \mathbf{T} & \mathbf{O} & \mathbf{I} & \mathbf{D} & \mathbf{E} & \mathbf{A} .\end{array}$}

Fig. 1 -5., 25. et 26. secundum Creplin Observat. de Entozois (no. 5). - Fig. 6-14. et 1\%. secundum Leuckart (no. 8). - Fig. 15. et 19. - 23. secundum Rudolphi (no. 14). - Fig. 16. secundum Zeder (no. 18).

Fig. 1. Caput Taeniae cucumerinae, rostello exserto.

a. Foramen in apice rostelli.

b. Canalis rostelli.

Fig. 2. Ejusdem caput, rostello ad partem solum exserto.

Fig. 3. Caput Taeniae tripunctatae.

Fig. 4. Caput Taeniae ellipticae, rostello toto recondito.

Fig. 5. Idem, rostello toto exserto.

a. Foramen in rostelli apice.

b. Canalis rostelli.

Fig. 6. Caput Bothriocephali planicipitis.

Fig. 7. Caput Bothriocephali Echeneidis.

Fig. 8. Capat Bothriocephali Floris.

Fig. 9. Caput Bothriocephali fragilis.

Fig. 10. Caput Bothriocephali Pilulae.

Fig. 11. Caput Bothriocephali Tricuspidis.

Fig. 12. Articuli Bothriocephali verticillati.

Fig. 13. Articuli Bothrioceplali Floris, evolnti.

a. a. Oraria, in eoram medio conspicua.

b.b. Filamenta alba, a Lenckarto pro genitalibus masculis habita.

Fig. 14. Articuli posteriores Bothriocephali Echeneidis.

a. a. Oraria, duas in scries juxta margines disposita, magna, nunc subrotunda, nunc oralia, colore brmescenti, translucentia.

Fig. 15. Pars Ligulae sparsae, magnitudine parum auctae:

a. Orarioram foramina impressa, trausrersalia, e quibus organa mascula, breria, teretia propendent, 
Fig. 16. Articuli medii Bothriocephali puetati.

Oraria hic utrinque regulariter sita et rerrucis simillima sunt.

Fig. 17. Articuli alii Bothrioceplali punctati.

Oraria in medio eorum conspiciuntur, forma irregulari ct situ variabili.

Fig. 18. Ora Taeniae crassulac, magnitudine ancta.
a. a. Chorion.
b. b. Amnion.
c. Foetus translucens.

Fig. 19. Ova'l'aeniae spluenoceplualae, pari modo aucta ac praecedentia. Fig. 20. Orum 'Tacniae longicipitis.
a. Chorion.
b. Allantoides.
c. Amnion.

Fig. 21. Orum Taeniae porosae.
a. Chorion.
b. Al!antoides.
c. Amnion.

Fig. 22. Orum Taeniae polymorphae.
a. Chorion.
b. Amnion orale.
c. Embryo linearis.

Fig. 23. Orum Taeniae crateriformis.

a. Chorion.

b. Amuion.

Fig. 24. Ovum Taeniae rariabilis.
a. Chorion.
b. Allantoides.
c. Amnion.

Fig. 25. et 26. Ora Taeniae cucumerinae, indusio pellucidissimo, pluribus commun, illclusa. 


\section{T A B. V. \\ $\begin{array}{llllllllll}\mathbf{C} & \mathbf{E} & \mathbf{S} & \mathbf{T} & \mathbf{O} & \mathbf{I} & \mathbf{D} & \mathbf{E} & \mathbf{A} \text {. }\end{array}$}

Secundum Bremser Ueber lebende Wïrmer im lebenden Menschen.

FIG. 1-8. BOTHRIOCEPHALUS LATUS. Ex intestinis tenuibus hominis.

Fig. 1. Pars antica, cum capite, magnitudine naturali.

Fig. 2. Caput, collo longo insidens, auctum.

Fig. 3. Capnt, collo brevissimo insidens, auctum.

Fig. 4. 5. 6. Articuli corporis dirersi, in quibus introitus ad genitalia, atque oraria tam foccundata, quam non foecundata, conspiciuntur.

Fig. 7. Articuli aucti, ita ut genitalia mascula clare in conspectum veniant.

Fig. 8. 0va matura.

FIG. 9-17. TAENA SOLIUM. Ex intestinis tenuibus hominis.

Fig. 9. Pars antica cum capite. Magnitudo naturalis.

Fig. 10. 11. 12. Caput auctum dirersis lateribus risum.

Fig. 13-17. Articuli dirersi.

In fig̣. 15. oraria dendritica translucent.

In fig. 17. articuli perforati sunt. 


\section{T A B. VI. \\ $\begin{array}{lllllllll}\mathbf{T} & \mathbf{R} & \mathbf{E} & \mathbf{M} & \mathbf{A} & \mathbf{T} & \mathbf{0} & \mathbf{D} & \mathbf{A} .\end{array}$}

Fig. 1-6. secundum icones originales Viri Exc. et Exp. Dr. Guilielmi Soemmerring, quas, nondum publici juris factas, ejus pater, Vir Illust. et Exc. Sam. Thom. de Soemmerring, summa mihi benevolentia communicavit. Fig. $z-9$. secundum tabulas, quas Viro b. Bremser jubente et auspice Vir Exp. Zehner ad vivum depinxit. Post mortem vero helminthologi illustris Vir Exc. et Ampliss. Profess. Fischer ex singulari sua humanitate earum me participem fecit.

Fig. 10 - 12. secundum Rudolphi Entoz. synosp. (no. 14.) tab. II.

FIG. 1-9. MONOS'TOHA FABA. Brems.

Parus major Viemnae in mercatu vivus cmtus et b. Bremsero allatus tubercula obtulit, quinque nimirum ad rentrem, duo vero in medio femore, quorum involucrum externum cutis tenera, aliquantum intensa, tantummodo exlibuit *). Tuberculorum unum et alterum Viro III. S. Th. de Soemmerring mittebatur, qui quidem, iis accuratissino examini subjectis, dissecando hanc structuram invenit.

T'ubercula forna globosa gandent, et magnitudine fructum Pisi sativi aequant. Formantur antem putamine $\mathbf{s}$, capsula duriuscula, elastica, superne foramine rotundo parro, quo partes infra describendae prominent, instructa. In cavitate capsulae corpuscula occurrunt duo, planiuscula, subhemisphaerica, forma fabis Coffeae arabicae s. lagenulis similia. Quorum corpusculorum, quae quidem vermes sunt, utrumque superficies offert duas, conrexam nimirum, et planam, planae faciei alterius corpusculi respondentem. Superficies modo commemoratae utrinque

*) Quod si lector de situ insolito horum animalculorum obstupescit, aliım, eumque multo magis mirum, domiciliandi Entozoorum modum, gratissima epistola a Viro venerabili Sam. Thom. de Soemmerring mihi communicatum, publici juris facio. Contigit nimirum Viro Exc. Dr. Cuil. Soemmerring (filio), testibus multis medicis Francofurti ad moenum habitantibus, Cysticercun vivum in puellae cujusdan camera oculi anteriore libere natantem observare. Parasitus nullum aliud symptoma, nisi, quando supra pupillae marginem assurrexit, inmedimentum risus, excitavit. 
marginibus conrexis, altro anterius, altro posterius sitis, describuntur. Inferiori parte margines sub arcus forma confluunt, superiori autem in extremitatem calyciformem, paululum reflexam (caput?) prolongantur. Caput locce, ex foramine capsulac supra dicto protrusum, undique liberum esse videtur, et penicili ope facile huc illuc movetur.

In superficic convexa, praesertim si lux a tergo in corpusculum resplendet, lineae conspiciuntur flexuosae, quae a capite inde ad extremitatem inferiorem serpentes, priusquam hanc attigerint, crancscunt. Quae rero striis caret extremitas punctis rariatur obscuris subrotundis. Lineac illae Soemmerringio partim canalis intestinalis, partim oraria pellucentia esse videntur. - In superficie contra plana nil commemoratu dignum reperitur.

Extremitas capitalis, a parte superiore accurate examinata, strias s. plicas, ex oblongo quodam spatio, os acquante, versus marginem ratliatim excuntes offert.

Ilace sunt, quae Soemmerring invenit. Observatis filii cura et arte delineatis adumbrationis excmplar, una cum verme uno ex capsula sua exemto, Bremsero misit.

Bremser *) e contrario in tuberculis, quae sibi retinuerat, nihil quod vermis speciem gerebat, reperire potuit, idque sine dubio accidit, cum, in capsulis ab ipso examinatis corpuscula jamjan exsiccata fuerint.

- Quae sequuntur ex literis hujus viri, Soemmerringio missis, desumsi, qui quidem summa benevolentia eas mecum communicavit, et in usum meum convertendi veniam dedit. Hac igitur renia utens, ex is ipsa verba huc pertinentia Viri scientiae, hen! nimis mature rapti, lingua vernacula typis exscribi aptum judicavi, gratumque lectoribus fore censui.

$$
\text { B r i e f s t e } 11 \text { e. }
$$

Tien, am 30. Juni 1823.

- - - - - - - - - - - - - - - - - - - „An dem hier geüfneten Balge war gar nichts einem Wurme ähnliches zu bemerken, (wovon ich auch jetzt die Ursache errathen kanu, die Würmer waren nämlich vertrocknet). - - _ - _ - In der vergangenen Noche wurde uns eine Tags vorher geschossene $S_{j} l-$ via sibillatrix eingebracht, die an beiden Kuieen, oder eigentlich Fersengelenken, älnliche $T u$. berkeln hatte. Ein Gelıülfe schnitt das eine auf, und brachte mir gleich daraus die in dem. selben enthaltenen zwei Würmer. Kanm hatte ich sie mit dem Suchglase einen Angenblick angeselıen, so langte ich in meine Tischlade, um Wilhelms Zeichnung herrorzuholen, und übergab sie ilım mit den Worten: IIier ist die Zeichnung davon. Indess beobachtete ich diese IVürmer aufmerksamer. Der Eine war etwas verletzt, und noch lebend, das hervorstehende hopfende $\Omega$ verwandelte sich bald in eine solche $\bigcirc \int$ Figur. Aber was sahe ich mun: der schwarze Sack, den Sie wegen Entfäbung durch Weingeist nicht lıben selıen können, wurde allmählig kleiner, und es schlïngelte sich allmälzlig der ganze Darm, mit 6chwarzem Koll gefüllt, ans dem Körper heraus, und endete mit einem weissen Punkte. Bei fortgesetzter Beobachtung sah ich wie der schwarze Sack sich wieder füllte, und der Darmhanfen, der aus. sen lag, runder wurde. Nach Verlauf etwa einer Minute war von den herausliegenden Dar. ıne, der sich auf der untern Seite wieder eingezogen latte, nichts mehr zu sehen, und der IVum schien ganz (integer). Indess war der Mahler aus dem zweiten Stocke herabgerufen worden, der die Tiürmer gleich lebend zeichnen sollte. Fiaum hatte ex sie betrachtet, so machte er die Bemerkung, dass aus dem einem sich wieder ein Wurm herauswinde. Nähere Untersuchung lelırte, dass es das Ilerauswinden des Dames war, der sich abermals ganz wieder zurückzog, und nun auclı so blieb. Eine wirklich merkwürdige Erscheinung. Die übrigen Tuberkeln wurden nun auch geöffıet, aber in den meisten waren die W'ürmer vertrocknet, doch hatten sie mehr die Form der eingesclickten Zeichnung $\Omega$. Ich habe nur zrrei Exemplare, 
Paulo post vero Sylvia sibillatrix telo uccisa scrutatori nostro eximio apportata est, quae in utroque articulo calcis ejusmodi tuberculis laborabat. 'Tubereulo aliquo dissecto, corpusculiscue exemtis Brenser vermes omuino non esse diversos ab illis, quorun delineationem paulo ante Soemmerring communicarerat, statim animadiertit. Uterque virus, alter autem paululum laesus fuit. Vermibus accuratius obserratis Bremser vidit extremitatem eapitis prominentcm, cujus ambitus modo angustus fuerat, in forman irregulariter rotundan veri. Nunc quoque in vermis interno sacculus quidam niger, secundum Bremseri opinionem canalis intestinalis, in conspectum venit. Jam vero sacculus gradatin, contrahebatur, et sensim scnsimque, nigris faecibus repletus, sub fo:ma fili, puncto albo exeuntis, ex toto prodiit. Quo facto, dictum saccum iterum volunine augeri, et acerrulum intestinorum extra corpus positum passu retrogrado minorem fieri observator vidlit, ita ut, sexagesima circiter lorae parte exacta, ne tantillum djuidem extra conspici posset, remis imo integer videretur.

Pictor quoque, ut vermes vivos delinearet, vocatus, oculis eos perlustrans, notavit, e corpusculo altero vermis quoddam simile proserpere, (quod revera canalis intestinalis fuit) et paulo post in caritatem se retrahens, quiete inclusum manere.

lieliqua tubercula, quae magis, quam exemplaria descripła (et fig. 7. - 9. efficta) formam figurarum a Soemmerringio missarum (fig. 1. - 6.) aequabant, pariter aperta sunt, sed in eorum plurimis rermes exsiccati fucrunt.

In tuberculis ceterum etiam a Bremsero constanter foramina, sed nunquam capita inde prominentia, qualia venerabilis Soemmerring ofiendebat, observata sunt.

Vir Excell. Fischer porro me literis hoc edocuit.

Tubcicula illa in Paro majori, Sylria sibillatrice, et Motacilla boanula huc usque prorenisse, et secundum ipsius obserrationes inter tubercula de variis arium generibus desumta non extare differentiam, tabulas potius Zehneri cum omnium partibus, in natura a se observatis, convenire.

Tubercula ant singula femoribus inhaerere, aut racematim juncta esse.

Vermem albicantem et pellucidum se prodere, ita ut struetura interna, qualis (fig. 7. - 9.) delineata sit, facili negotio cognosci possit.

Quoad diametrum latitudinem ipsi longitudine constanter majorem risam esse.

Bremserm hunc rermem, quia duo individaa uni capsulae constanti ratione inclusa reperiuntur, ab initio Monostoma geminum nominasse, ipsiusque animalculi effigiem in Icones helminthum recipere apud animum constituisse, postea rero, quia in Rudolphii scriptis hujus rer-

die übrigen guten und schlechten an Fudolphi geschickt. Die Oeffnung in den Tuberkeln ha. ben wir auch durclgängig gefunden, aber nirgends ausgestreckte höpfe. Die von mir verainstaltete Zeichnung werde ich Ihnen einmal zur Ansicht schicken. Auch eine Kopie von unsers Dohtors Zeichnung geht an Rudolphi ab, nebst dem Gutachten. Die W Ürmer heissen mir MIonostoma geminum. Als solche wurden sie gleich beim Anblick der Zeichnung taxirt, umd diese trifft sehr genau mit dem übersandten ausgelössten Würmlein überein; denn nun war die Untersuchung in einer Stunde beendiget. -

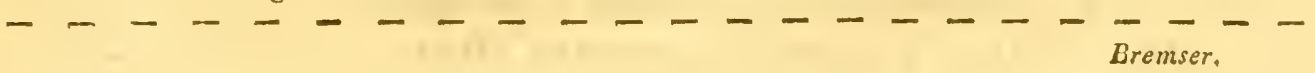


mis nondum mentio fit, illud consilium abjecisse, illa rero occasione alterum terminum, eumque qui nunc ralet, Monostoma Faba elegisse.

Nonnulla denique, quae a Soemmerringio in epistola mili scripta, hujus Entozoi causa monita sunt, lic afferam.

Egregius nempe vir nomen Monostoma rermi nostro minus accomodatum censet, monens eum cum rermibus generis Monostoma, in Bremseri Iconibus helminthum tab. VIII. fig. 1. - 12. depictis, nullam similitulinem habere. Monet porro titulo Monostoma neutiquan inesse vim, naturam vermis tantopere singularem significandi, additque hoc nomen magis accomodatum fore, si rermis forma gauderet plana, lingua simili. Itaque cum de genere omuino noro agatur, novum quoque uomen genericun Entozois nostris tribui necessarium, nec alium terminum Vir lllustr. aptiorem, magis significantem, magisque auctoribus optimis probatum judicat, quam tituJum ,Geminatus.“

Animum deinde adrertit nullum aliud extare exemplum vernium constanter tali modo binorum, dubitatque an forse unus horum rermium conjunctorun generis masculini, alter generis feminini sit.

Admonet denique exemplum binorum animalium ita inclusorum, ut neutrum se morere, nec locum unutare possit, huc usque non innotuisse.

Ipse multum operae, sed frustra, impendi, ut circa Dresdam ejusmodi rermes colligerem, eosque rivos dissecare possem, eoque magis me hujus roti participem fore sperareram, cum Vir Exc. et Exp. Thionemann, Prof. hist. nat. Dresdensis, simile tuberculum semel a se repertum fuisse, mihi asseruerit.

FIG. 1 -6. Fxemplaria in Paro majori inrenta:

FIG. 7-9. Eadein, in Sylvia sibillatrice reperta.

Fig. 1. 'Tubercula duo, magnitudine adaucta.

a. Ostium parrum, subrotundum, quo utrumque gaudet.

b. Caput ex illo libere prominens.

c. Pennae, tuberculis adluc inhaerentes.

Fig. 2. Eade1u, tuberculo uno dissecato.

a. a. Capita, libere prominentia.

b. Tuberculum dextrum dissecatum.

c. Lobus ejusdeni reflexus.

d. Corpora duo, fabis Coffece arabicae similia, sibi apposita.

Fig. 3. Vermis singulus, a superficie conrexa risus.

a. Puncta obscura, in ejus inferiore parte obrenientia.

b. Lineae, serpentiun ad instar flexae.

Fig. 4. I dem, a latere visus.

a. Superficies convexa.

b. Superficies plana, cui oppositus rermis adjacet.

Fig. 5. I dem, a superficie concava visus. 
Fig. 6. Caput, magis auctum.

a. Striae, s. plicae, a medio radiatim rersus marginem exeuntes.

Fig. 7. Vermis alter, adhuc rirus, a superficieconrcxa risus, magnitudine naturali.

Fig, S. I d c in, a da uctus.

Literarum explicationem vide sub descriptione figurae sequentis, quae quidem pro utraque icone ralet.

Fig. 9. Vermis, a superficie plana risus, adauctus.

a. a. Os.

b.b. Oesophagus, colore albido conspicuus, superne, ubi ab ore initium ducit, arctior, inferne dilatatus.

c.c. c. Organon quoddam inferiore partc bifidum, colorc fusco, canali intestinali Amphistomatis subtriquetri simile, (vide 'Tab. VIlI. Fig. 7. et 8 . Liter. f. g.) Haecce pars omnino canalis intestinalis rices gerere possit; si rero cuidam magis placeret organon varie flexum, litera (e.) (f.) notatum, pro dicto canali habere, illa forsan membrum hepati analogon judicanda est.

d.d.d. Organon, superne in extremitates duas latiores (semilunares) abjens, colore obscure flavo, punctis brunneis rariatum, cui non absque ulla ratione testiculorum titulus tribui possit.

e. e.f.f.f.f. Canalis quidam, superiorem reliquorum organorum partem rariis flexibus circumdans, coloris initio (e.) flaro-brunnei, dein rero (f.f.) nitide albi. Quod si hic ductus vas deferens, ex organo (d. d.) originem ducens, esset? Praeterea rero etian pro canali intestinali haberi potest.

g. g. Corpuscula alba, conglomerata, ad ductum quendam litera (h.) notatum racematim posita, in utraque rermis parte conspicua. Haec mihi ovaria esse videntur.

h.h. Vas, non minus album colorem prae se ferens, utrinque a modo commemoratis. corpusculis (g.) via transversa recia ad organon (i.) tendens, ex opinione mea ductus ouarionum efferens.

i. Corpus orale bullosum, colore lacteo distinctum, corpori orato Distomatis hepatici (vide Tab, VIII. fig. 3. et 4. lit. h.) simillimum. Receptaculum, in quod ductus ille efferens (h.) ora conducat, esse videtur.

k. $k$. Organon itilem coloris lactei, e resiculis singulis constans, quarum unaquaeque per ductum efferentem in ras medium commune abit, quod ipsum corpori ovali (i.) inseritur. Tesiculae forma oblenga, et ita positae sunt, ut pars tota folium plantarum umbelliferarum, sire molam resicularem aequet. Hoc organon vasi lactis albedine conspicno in Distomate hepatico reperiundo, et 'Tab. VIII. fig. 5. lit. I.I. delineato, simile, simili functioni inserrire milhi videtur.

1. 1.1. Pars quaedam, inter ramum alterum organi (d.) et inter partem (k.) obscure tantum conspicua, colore albescente, cui corpus orale insertum videtur, forsan pro utero habenda. 
m. m. Maculae duae albescentes, de quarum significatione nihil proferre audeo. Forma testiculis Distomatis lanceolati (vide 'Tab. VIII. fig. 12. lit. d.) respondent. FIF. 10. - 12. MONOSTONA WwULOLLE. E carnibus Lampridis guttati.

Fig. 10. Vermis a parte sinistra et inferiore risus, magnitudine naturali.

a. Porns terminalis, orbicnlaris, exiguns.

b. Collum teres, retrorsum attenuatum.

c. Corpus magnum, oblongum, apice ohtuso finitum, supra conrexum, subtus planiusculum, intus caritate vasis cutaneis praedita instructum.

d. Vas fuscum, rectiusculum per colli longitudinem medio currens.

e. Vasa in abdomine copiosa, flexibus Macandri more caudam rersus rergentia.

Fig. 11. I d em, magnitudiuc auctum.

a. a. a. Vasorum, in reme obrenientium, species prima. Fuscum ras, in abdomine ductus plurimos transrersos huc illuc flexos, irregulares, crassos, passim constrictos formans, quod Rudolphio (aeque ac pars in fig. 10. lit. d. notata) oviductus esse videtur.

b. b. Secunda rasorum pariter fuscorum species, ductus tenuiores, aequales, prioribus plurimam partem parallelos, passim vero illos decussantes, efficiens, secundum Rudolphii opinionem vasa scminifera.

c. c. Corpora majuscula, nuam daasre lineas longa, uunc oralia, nunc subrotunda, cum rasis crassis et irregularibus nexa. Otaria sunt, oris globosis minutis scatentia.

Fig. 12. Vermis portio ex abdomine desumta, eximic aucta, rasis triplicis ordinis optime distinguendis praedita.

a. a. Vasorum species prima. Oriductus.

b.b. Vasorum species secunda. Vasa seminifora.

c.c. Vasorum species tertia, rascula sistens tenuissima, albida, arctissimis retibus inter se conjuncta, in abdomine inter reliqua rasa occurrentia, in coilo rero frequentia, ut ejus tunicae fere inde contextae reperiantur. Quae quidem Rudolphio vasa nutritia esse ridentur. 


\section{T A B. VII.

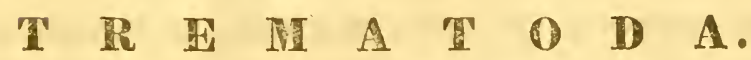

Fig. 1. - 3., 5.-9., 12. - 1\%. secundum Mehlis (no. 9.) - Fig. 4., 10., 11. secundum Bojanus (no. 1).

FIG. 1. - 10. DISTONA IIEPATICUM, Ex hepate et ductibus choledochis Otis Arietis, Boris Tauri etc.

Fig. 1. Distomathepaticum ejus magnitudinis, cujus in bobus plerumque est, a tergo risum.

a. a. Tybus cibarius ramosus, chymo fusco repletus.

b.b. Margines corporis, ab oris immaturis ibi accumulatis parum obfuscati.

Fig. 2. Idem, ab latere ventrali visum. 'Tubus cibarius inanis, hinc non conspicuus.

a. Acetabulum suctorium terminale.

b. Acctabulum suctorium rentrale,

c. Cirrus emissus.

d. Uterus perlucens.

e. Corpus oratum, albidum, ad quod

f. f. Ductus, ora ex orario efferentes, utrinque conreniunt.

ฐ. ฐ. Orarium, totum corporis marginem colore flavido insiguem occupans.

h. Media corporis pars, colore albido, rasa semen parantia continens.

i. Linea intermedia diaphana.

k.k. Rami majorcs ex summo trunco rasculorum dorsalium ad rentrem descendentes.

Fig. 3. Distomatis pars anterior, a dorso exhibita, magnitudine aucta. Cutis dorsalis ablata est.

a. Acetabulum suctorium terminale.

b. Appendix ejus, pro organo deglutitionis habenda.

c. Nerrus transrersus huic incumbens.

d. d. d.d. Intestinum ramosum, chymi fusci plenum.

e. e. Receptaculum penis.

f. Ductus uterque tenuissimus semen deferens huic adnexus. 
g. g. Uterus flexuosus, oris stipatus.

h. Corpusculum oratum, ex quo hic originem petit.

i. i. Ductus ex orario efferentes, qui ab utroque latere in corpus oratum conreniunt.

k. k. Orarii partes, inter marginales intestini ramos.

Fig. 4. Intestinum seorsim delineatum, ut forma ejus melius in conspectum veniat.

- Ilace figura secundum Bojanum (no. 1.) delineata est. Mehlis terminationes ramificationum ultimarum non acutas esse dicit, ut Bojanns exhibuit, sed clavarum in modum tumidas, ut fig. 3 , d. estendit.

Fig. 5. Pars distomatis anterior, a rentre picta, magnitudine aucta. Cutis ventralis usque ad ostia genitalia detracta est, una cum acetabulo suctorio posteriori.

a. Acetabuli terminalis ostium triangulum.

b. Penis exsertus, tortuosus.

c. Ostium genitale femineum, in quod

d. Capillus immissus est.

e. Uterus.

f. Ejus transitus ad porum femineum.

g. Ejus urigo ex corpore ovato.

I. Corpus oratum, albidum, carum, ora continens.

i.i.i.i. Ductuum ex orario efferentium pars longitudinalis.

k.k. Eorundem pars transtersalis.

1.1. Vas lactis albedine conspicuum, quod dextro plerumque corporis orati lateri adhaeret. Hoc inter cutem rentralem et tubum cibarium procedit, atque in ramos nounullos, ramulosque multos breves, apicibus magnis ampullaceis terminatos dirisum, spatio illo triangulari ad dextrum sito continetur, quod extrinsecus orario, introrsum et ante utero, retro vero transverso ovarii ducto efferente definitur. Frequenter ad ipsam insertionem constrictum reperitur. Lactei liquoris plenum est, quem ab ipso parari, et corpusculo orato modo commemorato adlatum excolendis ibi servire oris Cl. Melslisio probabile ridetur. Vide fig. 10. 0.

m.m.m.m. Orarium.

1.. Penis receptaculum.

o.0.0.0. Testis rasa contortuplicata, Canales sunt fere aeque ampli, extremis intestini ramulis magnitudine similes. Angustos in orbes torti, et summe multiplicibus circumducti flexibus, mire sibi implicati et adeo confusi sunt, ut erolri atque explicari nequeant. Circa lineam corporis mediam tamen magis paralleli sunt, et in

p.p. Truncos duos majores incidunt. Hi trunci rasculorum seminalium anfractibus obrolruntur et occultantur, atque semen colligere videntur. Alter, fere semper dexter, in parte posteriore; alter (sinister) anterius situs est. Trunci hi ad tres circiter lineas recti sunt, et in parte sua posteriori inter rascula complicata, (quorum nonnulla ipsis. imnittuntur) initium ducunt, in parte autem anteriori subito imminuuntur et transeunt in 


\section{$-19-$}

q. . Fila tanta tenuitate excellentia, ut absque lentis ope aegerrime discernantur, quae r'ursus in resiculam intra raginan penis latentem effunduntur, r. fig. 6. Hace fila humorem eundem lacteum, multis corpuseulis minimis, subsphaericis vel formac incertae, turbidum, qui in resicula membro virili adnexa reperitur, continent.

r.r.r.r. Intestini rami.

Fig. 6. Vesicula seminalis e corpore desumta, et receptaculo, quo continctur, liberata, simul cum pene, in quem transit, perfecte emisso, magnitudine magis, quam fig. praeced. aucta.
a. Penis (fere semper dextrorsum) arcuatus,
b. Apice distincte perforatus,
c. Basi cutis, ibi abscissae, reliquiis cinctus;
d. Receptaculi penis pars,
e. Vesiculac extremae adhaerens, in quam
f. Ductus semen deferentes aperiuntur.

Fig. 7. Eadem resicula, pene un emisso, pariter aucta.

Receptaculum penis, quo hae partes includuntur, plane remotum est, ipsaeque parum distractae et explicatae.

a. Cutis particula adhaerens.

b. Locus ubi penis emittendus foret. Foream format, atque in ea

c. Ostium femininum ad lacram conspicitur.

d. Uteri pars extrema.

e. Canalis, quo prolabente penis gignitur, tela cellulosa fibrosaque inrolutus.

f. Vesiculae extremitas.

Fig. S. Ovarii particula tenuissima, valde ancta.

Orarium componitur innumeris acinis, telae cellulosae inlıaerentibus, partin subsphaericis, partim magis oratis, magnitudiue varia, in universum vero ororum in utero contentorum ambitui similibus.

Unusquisque horum acinorum granulosus apparet, et velut minorum acinorum e congerie ortus, unde non orum, sed minimo:um ororum acervus esse videtur. Quisquis membrana involutus, rel sacculo inclusus esse videtur. IIos ororum globos urae racemorum ad instar vasculis_subtilissimis, qui inter ipsos perrcptant, adnexos esse vir Cl. Mel̄lis vidit.

a. a. Acini.

b. b. Vasa, quibus inhaerent.

Fig. 9. Cutis ventralis pars, ad demonstrandos nervos.

Ifuic parti acetabula suctoria, terminalis acetabuli appendix, receptaculum penis, et orariorum particulae tantummodo adhaerent, partibus reliquis sedulo remotis.

a. Acetabulam suctorium terminale.

b. Ejus appendix.

c. Receptaculum penis. 
1. Acetabulum suctorium posterius.

e.e. Orariorum partes.

f. Fila nervorum longa, quae ubi appendix acetabuli hoc ipsum attingit oriuntur, hine retrorsum et paululum extrorsum procedunt, tum media inter orarii margines et acetabulum posterius transeunt, tandem fere parallela et parum scrpentine flexa per corpus excurrunt, donec in cauda eranescant.

x. x. Lorundem extremitas, in ganglii modum tumida, ex qua reliqui nerri oriuntur.

g. Filum transversum tenue, quo extremae illorum filorum longorum partes (x. $x_{\text {. }}$ ), gangliis comparandae, inter se conjunctae sunt. Hoc proxine ab acetabulo suctorio' appendici ejus incumbit, annulumque dimidium format. (Alterum simile filum, collare complens, Mehlisius reperire non potuit.)

h. Filum nerreum, transicrso modo dieto tenuius, quod ex utroque summo nodulo (x.x.) codem loco quo illud inseritur exit, et rel juxta, rel supra acetabnlum procurrit. Hoc postquam parum intumuit in ramos dissolvitur ad acetabulum et cutem confinem adeuntes.

i. i. Filum nerreum, summe subtile et brevissimum, quod ex cujusris noduli parte externa egressum mox in cute delitescit.

Fig. 10. Partes genitales distomatis hepatici secundum Bojanum.

a. Porus anticus.

b. Porus rentralis.

c. Cirrus.

d. Corporis adumbratio.

c. Linea corporis media.

h. Pars oviducti anterior.

i. Pars ejusdem posterior, oris stipata.

k.l.m. Rami et ramuli orarii.

k. Rami transversales.

1.1. Rami longitudinales, in hos transeuntes.

m.m. Ramuli parri, quibus

p.p. Ororum germina, colore luteo, adhaerent.

o. 0 . Vesiculae ramosae.

FIG. 11. - 13. DIS'TOMA LANCEOLATUM. Ex corundem animalium ductibus biliariis.

Fig. 11. Secundum Bojanum. Distoma hepaticum junius, a latere ventrali, multum adactum.

a. Porus anticus.

b. Porus rentralis.

c. Cirrus.

d. e. Intestinum ramosum, bifidum (Mehlis hanc partem pro utero habet, $\mathbf{r}$ infra):

f. g. I. Corpora tria marginibus crenatis, quae Bojamus ad apparatum genitale fenineum refert, Mehlis vero pro testibus habet. 
i. :- Orarii inassa granulosa.

k. k. Lineae laterales lucidae, quas Bnjemus pro ramis Iongitndinalibus orarii habet, Mehlis vero pro ramis inlestini bifurcati, (ut in Amphistomate subtriquetro, v. tab, MIII. fig. 7. e.)

Fig. 12. Corporis media pars, ad illustrandum uteri et ovariorum il at uram.

a. a. Rami intestini bifurcati.

b. b. Oraria, colore lacteo insignia, ramosa, quac acinis, nunc racematim dispositis, nunc in lineas junctis, composita videntur.

c. c. Ductus ora efferentes, ex asteriori et interua eorun parte proficiscentes.

d. Macula subrotunda, in rua hi ductus conveniunt. Haec constanter subrotunda, et nunqnam testium trium anteriorum in modum; lobata est, minusque is perlucet, nec ut iidem contextus acinosi speciem praebet, Corpori orato Bistomatis hepatici (r. fig. 3. h.) analogon est, et ad idem officium ac hocce comparata videtur.

e. e. Uterus. Hic tubus simplex amplus est, qui primo retrorsum caudamque rersus procedit, jam rero ibi replicatus recurrit, et denique proxime locum, quo penis exscritur, in porum femineum externum exit. In hoc cursu modo ad hoc, modo ad ilind latus defiectitur, modo in medium corpus redux est, et sic maxime multiplicibus anfractibus inter tubi cibarii crura circumit. $\mathbf{A b}$ ororum, quibus paene nunquam non stipatus est, colore tinctus est, ita ut omnes ab albo usque ad fuscum colores induat, extrema rero ejus pars plerumque rel pauca rel nulla ora continet.

Fig. 13. Corporis particula, antecedente magis aucta, ad repraesentandum modum, quo tubi cibarii crura in cauda finiuntur.

a. Uterus.

b. 'Tubus cibarius.

FIG. 14. - 18. DIVERSA DISTOMATUM OVA, MICROSCOPII COMPOSITI OPE AUCTA.

\section{FIG. 14. - 17. DISTOHATIS HEPATICI,}

FIG. 1S. LANCEOLATI OVA.

Fig. 14. Ora immatura, ex ovarii ducto efferente.

Fig. 15. Ora in corpusculo orato aliquando reperta.

Fig. 16. Ora maturescentia, qualia in uteri parte corpusculo orato proxima occurrunt.

Fig. 17. Ora matura, quae utero extremo insunt.

Fig. 18. Ora Distomatis lanceolati.
a. Ex uteri initio, colore albo.
b. Ex uteri media parte, colore flaro.
c. Ex uteri extrema parte, colore fusco. 


\section{T A B. VIII.}

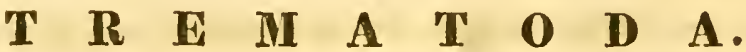

Fig. 1. - 3. secundum Jurine (no. 7). - Fig. 4. - 10. secundum Bojanus (no. 1). - Fig. 11.-16. secundum Nitzsch (no. 10).

FIG. 1. - 3. DISTOHA LUCII. Ex intestinis Esocis lucii.

Fig. 1. Vermis, a laterevisus, magnitudine naturali.

Fig. 2. Idem, ab inferiori, magnitudine adauctus.

a. Porus anticus.

b. Portis rentralis.

c. c.c. Canalis intestinalis:

d. Saccus ejusdem coecus.

e. Ejusdem canalis sphincter, qui a poro antico initium foramine parro ducit.

f. f. Filamenta nervosa, quae inde a poro antico partem internam utriusque rani canalis intestinalis sequuntur, et pone porum posteriorem ad corporis partes interuas distribuuntur.

g. g. Orarium, ovis repletum.

h. Oviductus, qui foramine parro aperitur.

i. Globuli tres, sphaerici, colore albo, pone orarium situati.

Fig. 3. Idcm, cadem magnitudine, a superiori.

b. Porus rentralis a posteriori.

c. c. Canalis intestinalis.

d. Ejusdem saccus coecus.

g. Orarium.

h. Oriductus, oris repletus:

i. Globuli tres, Ab horum primo s. antico oritur

k.k. Vas albicans, leviter undulatum, quod versus corporis ntrumque latus prodit, et ilji in ramos se findens encarpa lutescenti-alba format.

FIG. 4. - 10. AMPHISTOMA SUBTRIQUETRUM. Ex intestinis crassis Castoris Fiberis. 
Fig. 4. Vermis ab inferiori. Magnitudo naturalis.
a. Porus anticus.
b. Porus genitalis.
c. Porus ventralis.

Fig. 5. Idem a latere visus.
a. Porus anticus.
b. Ports genitalis.
c. Porus rentralis, crijus fundus in rerrucae formam propulsus, et perspicue non per- foratus est.

Fig. 6. Vermis a dorso, auctus.

Conspiciuntur fibrae musculorum, triplici directione decurrentes.

Fig. 7. Amphistomatis interna, a rentris latere visa. Vermis in me. dio abdomine longitudinaliter ita sectus est, ut porus ge. nitalis integer, una cum cute, dextrorsum revolutus sit.

a. Porus anticus, integer, qui in canalem intestinalem conducitur.

b. Locus, ubi genitalia ad porum genitalem transeunt.

c. c. Porus rentralis, medio dissectus, nou perforatus.

d. e.f. æ. g. Tractus intestinalis, canalem bifidum formans.

d. Ejus pars, inde a poro antico in pharyngi modum amplificata.

e. Canalis angustus, oesophago analogus.

f. Locus, ubi in duos ramos utriformes finditur.

g. g. Uterque saccus coecus, quo finitur.

h. i. k. I. l. Genitale masculum.

h. Cirrus, nonnumquam e poro genitali prominens.

i. Bulbus ejusdem crassior et torosus, ex quo

k. Duo rami (ductus deferentes), orarium amplectentes descendunt, quorum uterque pertinet ad

1. 1. Corpora fascicularia (resiculas seminales), quorum singulum ex quatuor paribus canalium dichotomorum, quae conjunctae sedecim terminationes coecas habent, compositum est.

m. n. Oriductus, oris repletus.

m. Fundus ejusdem.

n. Extremitas, transiens ad porum genitalem.

o. o. Massa granulosa, ororum germina.

p. q.r.s.t.u. Systema nervosum.

p. Ganglium planum, utrinque ad oesopliagum situm;

q. Filum, inter utrumqne ganglium transrersum.

r. Fila tria, ex ganglio antrorsum exeuntia.

s. Fila, ex eo postrorsum exeuntia, ex qnibus prodit

t. Filum alterum transrersum, in abdominis latere situm, (collare):

u. Fila postica, ad intestina et cutem pertinentia. 
Fig. 8. Amphistomatis interna, a laterc dorsali visa, cute incisa et ieposita.

Literae idem, quod in figura praecedenti, denotant.

Perspicue conspicitur filum (o.) inter ganglia (p.) transtersum.

Fig. 9. Genitalia, multum adaucta.

b. Locus pori genitalis.

b. Cirrus.

i. Bulbus ejustem.

k. k. Ductus deferentes.

1.1. Corpora fascicularia.

m. Fundus oriductus, oris repletus.

11. Extremitas cjus.

o. Massa granulosa, ovorum germina;

Fig. 10. Ora maturitatis dirersae, multum adacta.

a. Orum pellucidum.

b. Orum puncto obscuro rersus extremitatem instructum.

c. Orum medio puncto obscuro notatum.

FIG. 11. - 16. HoLOSTONUM SERPENS̃, Nitzsch. Ex intestinis Falconis Haliaëtos.

Fig. 11. Vermes magnitudine naturali.
A. A. А. Pars corporis anterior.
B. B. B. Pars corporis posterior.
X. Individua duo coitu juncta.

Fig. 12. Vermis magnitudine auctus.

Intestina translucent.

A. Pars corporis anterior, breris.

B. Pars corporis posterior, longa et tenuis.

a. Os.

b. b. Tentacula duo rel laciniae.

c. Superficies rentralis cara.

f. Duo paria laciniarum excaratarum, a prominentia, in superficie cara rentrali sita, originem ducentia.

g. Apertura genitalium.

h. 'Testiculi duo, culeo inclusi, quorum alter posi alterum situs est.

i. Orarium.

k. Ora.

Fig. 13. et 14. Pars corporis anterior, oris instar contracta, a latere vis a.

c. c. Superficies ejus rentralis cara, rerum os recondens.

f. f. Duo paria laciniarum inde prominentia.

1.1. Superficies dorsalis. 
$-25-$

Fig. 15. Pars corporis anterior, a ventre $r$ isa.

a. Os.

b. b. Tentacula duo.

d. Prominentia in superficie corporis rentrali.

e. Duo palpi, antice inde exeuntes.

f. Laciniae excaratae, postice inde prodeuntes.

Fig. 16. Pars corporis anterior, a tergo risa.

b. b. Tentacula duo.

f. Laciniac excaratae, a prominentia supcrficiei rentralis orientes. 


\section{A C A N T H O C E P HA I A.}

Secundum Jules Cloquet Anatomie des rers intestinaux: Ascaride lombricoide, et Echinorhynque géant (no. 4).

ECIIINORHYNCHUS GIGAS. Ex intestinis tenuibus Suis Scrophae.

Fig. 1. Mas, magnitudinc naturali.

Vermis mortuus in aqua immersus, eaque firmus, cylindricus, et clasticus factus est, cum in statu viro applantus, mollis et rugosus fuerit.

a. Proboscis.

b. Collum.

c. Cauda.

d. Organum genitale, sub forma campanulae, cujus pediculus apici caudae adhaeret, externe prominens.

Fig. 2. Idem mas, a laterc abdominis, longitudinaliter fissus.

a. Proboscidis caput, cun uncinulis.

b. Proboscidis pars, in corpore inclusa,

c. Musculi retractores proboscidis.

d. d. Lemnisci.

e. c. Canales laterales.

f. 'Testiculus anterior.

g. Canalis, inde ab hoc organo usque ad extremitatem proboscidis posteriorem pertinens.

1. Testiculus postcrior.

i. i. Canales defercntes duo, a parte posteriore testiculorum orti.

I. Canalis unicus, ex conjunctione duorum praecedentium ortus.

k. Dilatationes canalis defcrentis, resiculam seminalen formantes.

m. Penis, resiculae seminali succedens.

11. Carum penis conoideum anterius. 
o. Carum penis conoideum posterius. Basis utriusque conjuncta est.

p. Caudae apertura.

q. Rosula per initium penis formata, co loco ubi ille cum resicula continnatur.

r. r. Musculi retractores penis,

s.s. Musculi protractores penis.

Fjg. 3. Femina, a latere abdominis, longitudinaliter fissa. Maguitudo naturalis.

Ovarium abdominale in tota sua longitudine apertnu est, oris in cocontentis remotis, ita ut solummodo ea orari dor. salis reliqua sint.

a. Caput proboscidis, externe visibile.

b. Proboscidis corpus, in caritate abdominis latens.

bb. Musculi protractores proboscidis.

c.c. Musculi retractores proboscidis.

d. d. Lemuisci.

c. c. Canales longitudinales.

f. f. Fibrac musculares longitudinales corporis.

g. g. g. h.h. Orarium superius s. dorsale, continens ova, in cylindros passim interruptos unita, et eo magis roluminosa, quanto propriora extremitati corporis anteriori sunt.

i. Ovarii dorsalis apcrtura in abdominale ovarium.

k. Sacculus coecus, a communi ovariorum cavitate ad collum formatus priusquam haec proboscidi inscritur.

1. Finis orarii dorsalis.

m. Canalis parietibus albis crassis instructus, quo orarium abdominale terminatur, (oviductus).

\section{FIGURAE SEQUEN'TES MICROSCOPIO AUCTAE SUNT.}

Fig. 4. Extremitas anterior Echinorhynchi, proboscide egressa.
a. Tuberculum centrale in apice proboscidis conspicuum.
b. Pediculus, quo eminentia proboscidis globosa suffulcitur.
c. Sulcus, hunc pediculun a collo separans.
d. Collum.
f. Unciudi, quibus caput munitum est.

Fig. 5. Eminentia proboscidis globosa, ab anteriori visa.

Mcdio tuberculum centrale, et porus s. apertura proboscidis, nec minus uncinulorum dispositio in quincunce conspicitur.

Fig. 6. Unus ex uncinalis proboscidis a latere visus. Multum ada u ctus.

a. Ejus apcx.

b. Basis in eminentia globosa proboscidis recepta, singulariter inflata. 
Fig. 7. Extremitas anterior corporis, longitndinaliter aperta.

Orarii ad proboscidcm prolongatio resccta est, cujus musculi melius in conspectum reniant.

a. Caput proboscidis.

b. Corpus ejusdem amplexum per

c. c. Duos musculos protractores proboscidis, ab una parte apici colli, ab altera extremitati moboscidis posterioni affixos.

d. d. Duo musculi retractores proboscidis superiores, (ab invicem remoti, sectione corporis inter $\cos$ facta).

e. e. Duo musculi retractores proboscidis inferiores.

f. f. Lenuisci.

Fig. S. Extremitas anterior corporis longitudinaliter secta.

Proboscis in corpus retracta et in se ipsam ita inversa est, ut exterua cjus facies interna facta sit, et vice rersa.
a. Integumentum corporis.
b. Integumentum colli.
c. Eminentia globosa prohoscidis in se inrersa, ita ut uncinuli interne reperiantur.
d. Corpus cylindricum proboscidis ad partem fissum, ita ut caritas centralis conspiciatur.
e.c. Musculi protractores.
f. Musculi retractores superiores.
g. Musculi retractores inferiores.
l., h. Lemnisci.

lig. 9. Scctio transrersalis feminae, in parte corporis media facta.
a. Pellis.
b. Duo strata muscularia, et substantia pulposa subcutanea.
c. Canalis subcutareus dorsi.
d. Canalis subcutancus abdominis.
c.e. Canales latcraies.
f. Caritas orarii dorsalis.
g. Caritas ovarii abdominalis.

h. h. Siatia triangularia inter oraria et corpus, in quibus canales laterales prominent.

i. Septum transrersum oraria discerucns.

Fig. 10. Extremitas caudac naris, organo genitali externo plane in abdo. men retracto.

a. Fissura lincaris, Iabiis tenuissimis, ad caudan extremam sita.

Fig. 11. Portio eadem.

Fissurae labia postrorsum torta, rotundata et prominentias unt.

Fig. 12. Eadcm extremitas candac maris longitudinaliter fissa. Magis aucta.

a. a. Integumentum corporis exterius, perspicue continuatum cum 
b. b. Membrana externa organi genitalis, conum formante posteriorem, qui simulac per fissuram prodiit, exterior fit.

c. Caritas conica organi genitalis, obducta per comum anteriorem, qui intravit posterio. rem, et continuatur cum

d. Caritate penis, longitudiualiter resecta.

e. Locus, ubi uterque conus unitur.

f. Spatium, inter duos conos restans, (quorum unus, ratione modo illustrata, alterum in se recipit), et cum cavitate abdominali communicans.

g. g. I'ortio musculorum protractorum penis.

Fig. 13. Ambooraria Echinorhynchi, ex corpore deprompta, magnitudine uaturali.

orarium abdominale antrorsum conspicitur, et ad partem apertum est.

a. Proboscis.

b. Canalis, quem caritas orariorum communis (saccus coecus) ad proboscidem emittit.

c. Caritas orariorum communis aperta, ita ut ejus interins in conspectum reniat.

d. Aditus ad saccum coecum per hanc cavitatem formatum.

c. Orarii dorsalis apertura in abdominale, ad extremitatem septi comıntuis, quod margine

- concaro, post hanc aperturam conspiciendo, terminatur.

f. f. Ora in orario abdominali contenta.

g. g. Ora in ovario dorsali contenta, et septo communi a praecedentibus separata.

h. Saccus coecus, per finem posteriorem orarii dorsalis formatus.

i. Oviductus s. canalis quo orarium abdominale postice terminatur.

Fig. 14. Extremitates posteriores utriusque orarii, a latere risae. Mul$t$ um a d a uctae.

a. Saccus coecus, per orarium dorsale formatus.

b. Extremitas orarii abdominalis posterior, cohaercus cum

c. Oviducto.

d. Insertio hujus canalis in caudam mediam.

e. e. Integumentum corporis.

Fig. 15. Extremitas caudalis feminae, amplexa et ornata per corpus fig. 16. deline a $t$ um.

a. Cauda.

b. Corpus riride.

Fig. 16. Corpus conicum carum, coloris viridis, quod extremitaticaudi diversarum feminarum adiacrens a Viro Clarr. Cloquet reperitum est, a latere risum.

a. Aper.

b. Cavitas, caudam excipiens.

c. Macula alba, in fundo conspicienda, 


\section{$-30$}

Fig, 17. Idem corpus ab anteriore.

Humor lacteus quem illud media parte (a.) contincbat, late expansum est, et

b. Immensa quantitate oxulorum maxime tenuium, pellucidorum, oblongorum, embryone destitutorum, formatur.

Fig. 18. Ova pellucida, non foecundata, quac in orariis oris sequentibus immixta reperiuntur.

Fig. 19. Ora foccundata, in quibus embryo, utplurimum centralis, forma multum variabili, observatur.

Fig. 20. Eadem ora, magis evoluta. Opaca in ambitu et pellucidain centro sunt.

Fig. 21. et 22. Ora, quac ad maturitatem pervenerunt. 


\section{T A B. X.}

\section{A C A N T H O C E P H A I.}

Fig. 23. secundum Jules Cloquet (no. 4). - Fig. 24. - 28. secundum Bojanus (no. 1). - Fig. 29.-36. secundum Westmub (no. 16.)

FIG. 23. - 32. ECHINORIIYNCIUUS GIGAS. Ex intestinis tenuibus Suis Scrophae.

Fig. 23. Duo Echinorhyuchi in coitu.
a. Portio intestini ilei aperti.
b. Corpus feninac.
c. Mas, proboscidis ope, in intestino fixus.
d. Organum genitale maris, amplectens et in caritatem suam recipiens
e. Extremitatem candalem feminac.

Fig. 24. Portio tunicae muscularis, ab interno, multum adaucta.

p.p. Stratum interius, per fibras longitndinales dense positas formatum.

q. ฯ. . Stratum exterius, per fibras circulares, in fasciculis ab invicem remotis positas, formatum.

Fig. 25. Sectio maris transversalis, in regione testiculi inferioris, qui ipse sectione attingitur.

g. Testiculus perscissus.

h. Stratum musculare externum.

k. Stratum musculare internum.

1. Canalis dorsalis.

m. Canalis abdominalis.

u.x. Carum abdominis duplex.

y.y. Prominentiae in parietibus lateralibas, minus regulares quam in femina.

z. Scptum transrersum, quo abdomen longitudinaliter in duo cara (u. x.) separatur, quorum unum ( $\mathrm{x}_{\text {. }}$ ) testiculos recipit, alterum $\left(\mathrm{u}_{\text {. }}\right)$ autem racuum est.

Fig. 26. Sectiofeminactransversalis, in extremitatc superiore, ex regione, usque ad quam lemnisci pertinent.

g. g. Lemnisci perscissi.

h.h. Cutis externa. 
k. Tunica muscularis.

1. Canalis dorsalis.

m. Canalis abdominalis.

p. Cavum orarii.

x.x. Spatium inter parietem lateralem corporis et parietem membranceum orarii utrinque residuum, in quo lemuisci jacent.

Fig. 27. Extremitas anterior feminae, $10 n g i t u d i n a l i t e r f i s s a c, a d a u c t a$.

a. Proboscis.

b. Vagina ejus, ita praeparata ut libere conspici possit.

c. Musculi protractores proboscidis superiores.

d. Musculi protractores proboscidis inferiores.

e. Musculi retractores proboscidis superiores.

f. Musculi retractores proboscidis inferiores.

g. g.g. Lemnisci.

h.i.k. Triplex stratum cutis, sectione visibile.

l. Cutis externa.

i. Stratum medium flarum.

k. Stratum internum, s. membrana muscularis.

1. m. Aperturae canalium longitudinalium.

n. Vestigia linearum longitudinalium obsoletarum. Ifae lineae nsque ad extremitatem rermis anteriorem non delineatac, sed tantummodo leviter adumbratae sunt, distantiis ab invicem tamen sedulo observatis.

o.p. Ovariun.

o. Ejus initiun tenue et filiforne a fundo proboscidis.

p. Ejus pars dilatata, cui ova insunt.

Fig. 2S. Extremitas posterior feminac, discissa.

q.r. Extremitas ovarii inferior.

q. Pars ejus ampla.

r. Pars coarctata.

s. Prominentiae globosae (resiculae), inter quas orarium inseritur.

t. t. Duo fasciculi floccosi, pediculorum ope resiculis (s.) adhaerentes, ororum expertes.

u. u. Duo sacculi oblongi, cum iisdem resiculis (s.) cohacrentes.

จ. Vagina, ova continens.

w. Ostinn raginae in extremitate corporis.

Fig. 29. Partes feminac sexuales c corpore depromptae. Multam auctae.

a. Uterus, in infima cauda situs, forma oblongo-acuminatus. In apice superiore abit in

b. Duo parra cornua, ex quibus

c. c. Dito ductus angusti oriuntur, qui com

d.d. Orario bipartito placentulis repleto comınunicant.

e. e.e. Ligamentorum trium tendinoso-cellusorum ope hae partes cuti adhaerent. 
Fig. 30. Mas in statu evolutionis. Magnitudo naturalis.

a. Proboscis retracta.

b.b.b.b. Ejus musculi protrusores et retractores.

c. Lemnisci conroluti.

d. Testiculus anterior.

e. Testiculus posterior.

Hi testiculi duabus ex membranis formantar, quarum extcrna crassior et durior, interna tenuior et mncosa est, quaeque ambae parenchyma vasculosum et grumosum includunt. Praeter has rero membranas; tunicas testiculorum proprias facientes, tunica etiam cellulosa viaphana, a cute interna exorta, illos ambit, sicut quoque hinc illincre fila musculosa conspicua sunt. Membrana haec cellolosa rersus anticam partem folliculum efficit, per cujus medium ras (f.) ad raginam proboscidis pergit, quod musculis protrusoribus membranisque raginae perforatis, ostio minutissimo in vaginae (a.) carnun hiat. Vas hoc, arteriac spermaticae analogon, parenchyma testiculi superioris perreptat, et hoc absolato (g.) aequali magnitndine interiorem testiculum intrat.' 'Testiculus inferior nunc hnjus vasis (li.) ope, cum ipsis resiczlis scminalibus conjunctus est.,

f. Vas ad ragiumm proboscillis usque pertinens, arteriae spermaticae analogon.

s. Pars ejusdem, testiculis intermedia.

h. Pars, qua testiculus inferior cum resiculis seminalibus conjungitur.

i. Vesiculae seminales.

k. Musculi ejusdem.

1. Ductns, penis rices gerens, muscalis propriis instractus.

Fig. 31. Lemniscus Echinorhynchi recentis. Auctus.

Uterque lemniseas duornm rel plurinm filorum ligamentoso-rasczlosorum ope in sito suo retinetur, apice vero in ipsius raginae proboscidis caram spectat.

a. Pars eornm superior, angustata.

b. Pars inferior, latior, libere in caro corporis fluctuans.

c. Basis libera, rotundata.

In illis lentis fortissimae ope tria rasa magna (d.e.f.) in conspectum reniunt, quorum medium idque maximum (e.) in medio lemmisco, reliqua duo (l.f.) utroqne in margine ejus sita sunt. Medium ras, in lemnisci antica et summa parte exortum, per tres quartas ejus partes recta ria profectum, et in itinere suo ramis in infinitum iterum ramificatis enissis, sacculum magnum oralem (s.) intrat. Ex hoc sacculo vas aequali amplitudine continuatur, et rersus extremum lemniscum in duos ramos, açque magnos (h. i.) sub angulo acuto finditur, qui iterum ramos ramulosque rarios edentes usque ad basin lemnisci snbstantiam perreptant, ibique inflexi rel eum vase utroque in margine conspicuo (d.f.) anastomosin formant (k.k.), rel in his ad apiccm recurrunt. Sacculus modo dictus non unicus antem in lemnisco conspicitur, sed rami ramulique undique anastomosantes sacculos parros et minutos, dilatationes rasorum coëuntium scilicet, formant, et in superiore parte quinque rel sex minores, rariae formac, trigoni, inaequales 
apparent, quorum duo (1.1.) in lemnisco delineato utroque in margine, duo altera (m. m.) cum rase magno eoque medio communicantes obserrantur.

FIG. 32. ECHiNORHYNCHUS HALRUCA. Ex intestinis Ranae esculentae.

Pars membranae secundae, vasculosac, quae cpidermidem et musculorum stratum cxterius interest, cui particula epidermidis adliuc adliaeret.

Hace membrana, quoad structuram mucosa, plerumque flacescens, myriadibus rasorum anastomosantium perreptatur, quorum rami principales ex reti utroque in latere longitudinaliter disposito, (ac verme dissecto in interna facie ita conspicuo, ut inter fibras musculares longitudinales ct circtiares intertextum sit) exortum ducunt. Retium horum raıi undique coëuntes anastomoses formant, quae majores adluc hac in membrana mucosa existunt, ut fere tota rasis constituia sit, et cx quibus denique ramis serpentium ad instar membranas percurentibus nonnulli ipsis cum poris communicare videntur.

FIG. 33. et 34. ECHINORIYNCHUS MAJOR. Ex intestinis Erinacei europaei.

Fig. 33. Femina, longitudinaliter aperta. Magnitudo naturalis.
a. Proboscis.
b. Ejus ragina.
c. Lemnisci, nodulosi, longitudine inaequales.
d. Ovaria ambo.
e. Uterus.

Fig. 34. Ejusdem pars antica, multum adaucta, ita ut lemniscorum forma et raginae proboscidis musculi in couspectum reniant.
a. Proboscis.
b. Vagina ejus,
n. Musculis suis protrusoribus et retractoribus instructa.
c. Lemnisci nodulosi. (Vas medium quod, ab uno nodulo ad alterum percurrens, un- dique ramos in substantiam emittit non delineatum est.)
d. Duo oraria.

FIG. 35. et 36. ECHivorHyncirUs CAUDATUS. Ex intestinis Falconis.

Fig. 35. Mas, erolutus, longitudinaliter sectus. Magnitudo naturalis.
a. Proboscis.
b. Ejus ragina.
c. Musculi.
d. Lemnisci, breres, complanati.
e. T'esticuli, qui anticam corporis partem occupant, et quorum unus alteri incumbit.
f. Vas defcrens.
g. Vesicula seminalis.
h. Musculi ejusdem.
i. Organon masculum, protrusum. 


\section{$-35$}

Fig. 36. Femina, longitudiuliter secta. Magnitudo naturalis,
a. Proboscis.
b. Ejus ragina.
c. Ejus musculi.
d. Lemuisci ambo.
c. Oraria.

f.f. Placentulae in orariorum parte anteriore contentac,

g. h. Uterus.

ฐ. Pars ejusdem stiperior oralis, cum orario comınuncans.

h. Pars inferior tumidala, attenuato-oralis, 
T A B. XI.

\section{A C A N T H O C E P H A I A.}

Fig. 1. $-8^{\mathrm{b}}$, et fig. $9-14$. secundum Westrumb de helminth. acanthoceph. (no. 16.) - Fig. $S^{\text {b }}$ secundum Rudolphi Ent. hist. natur. (no. 13.)

FlG. 1-6. ECIINOHHychus PRoteus. Ex intestinis Cyprini Barbi.

Fig. 1. Exemplar collo subaequali.

Fig. 2. Pars veruis antica, ubi collum anterius bulla terminatur.

Fig. 3. Mas, apertus.

a. Proboscis.

b. Bulla colli.

c. Colli pars subconica.

d. Vagina proboscidis.

e. f. 'Testiculi conjuncti, in antica corporis parte siti.

g. Vesicula seminalis, in fig. 5 . eroluta.

h. Ductus, vesiculae insequens.

i. Tubulus acuminatus, penis vices gerens.

k. Lemnisci.

Fig. 4. Femina aperta.

a. Proboscis.

b. Bulla colli.

c. Vagina proboscidis.

d. IIujus musculi.

e. Lemnisci.

f. Orarium placentulas continens.

g. Uterus.

Fig. 5. Vesicula seminalis, ex corpore deprompta et evoluta.

a. T'esticulus posterior.

b. Diverticula, quibus vesicula seminalis componitur, sejuncta, sell

c.c. Vasorum parrorum ope inter se conjuncta. 
Fig. 6.a. Uterus ex femina depromptus.

a. Orarium placentulas continens.

Fig. 6, b. Placentulae, ex orario.

Puncta rotunda vel oralia, quae in iis conspiciuntur, forsan orula immatura sunt, quae maturiora facta his placentulis solummodo apicis auxilio inhaerent, donec ipsum uterum intrent.

Fig. 6.c. Ora matura, ex utero, forma lineari.

FIG. 7. a.b. ECHNORHYNCIUS MACRACANTHUS. Ex intestinis Charadrii phutalis.

Fig. 7. a. Exemplar integrum, adauctum.
a. Proboscis magna, subglobosa.
b. Papilla suctoria.
c. Collum brere, raginatum.
d. Corpus oboratum.

Fig. 7.b. Papilla suctoria proboscidis.

a. Striae radiiformes, a Viro Clarr. Westrumb pro rasis absorbentibus habitae.

FIG. 7. с. ECHINORHYNCHVS POLYMORPIUUS.

Bulla senilis vermis ab rortice inspecta, ita ut papilla in conspectum reniat.

a. Striae rasculosae, ramis abeuntibus, totamque bullam perreptantibus.

FIG. 8. a. ECHiNorhycilus COnPRESSUS. Ex intestinis Corvi Picae.

Apex caudalis speciminis unius notatu digui, vesicula genitali terminatus.

Lentis ope resiculae cyathiformis (a.) superficies interna undique punctis opacis teretibus s. triangularibus, inaequalibus adspersa conspicitur. Pars resiculae antica utroque in latere resicam parvam lagenae ad formam (b.) emittit, et ex apice stylus opacus (c.) exsertus est, qui mediam partem resiculae legens, ex corpore ipso erumpit.

FIG. 8. b. ECHNoRHXNCHUs Nodulosus. Cyprini timbae.

$$
\text { Pars inferior rermis, aucta. }
$$

a. Corporis pars inferior.

b. Sacculus huic annexus.

c. Globuli ex eodem prominuli.

FIG. 9. ECHNORHYNCIUS MONILIFORMIS. Muris arvalis.

Exemplar integrum, magnitudine naturali.

a. Proboscis minutissima,

b. Corporis pars anterior, stricturis transiersis intercepta, quae ita formantur, ut musculorum externorum s. annularium fibrae in fasciculis dispositac sint, et ubi rermis constrictus est maxime firmi et densi appareant.

c. Corporis pars posterior, teres. 
FIG. 10. ECHINORHYNCHUS CAUDATUS. Falionis Buseonis,

Mas integer, adauctus.

a. Proboscis.

b. Corporis pars crassior.

c. Ejusdem pars tenuior.

d. Vesicula caudalis.

FiG. 11. ECHINORHÝNCIIUS PLAGICEPHALUS. Acipenseris Hasonis.

Pars antica, adaucta.

Proboscis longissima clarata, panilla suctovia prominente, collum brerissimum.

FIG. 12. ECHINORHYNCIIUS PYRIFORMIS. Turdi Meruiae.

Exemplar integrum, auctum.

a. Proboscis clavata。

b. Corporis pyriformis pars antica, crassissima, snbglobosa, aculeîs exignis dense armata.

c. Corporis pars postica, conice decrescens.

FlG. 13. ECIINORHYNCIUS SPIAEROCEPIALUS. Hinantopostis ostralegi.

Exemplar junius, adactum.

a. Proboscis globosa.

b. Collum longum, subconicum, laere.

c. Pars corporis anterior, aculeata.

d. Pars corporis posterior, nuda.

e. Vesicula caudalis.

FIG. 14. ECHINORHYNCHUS HYSTRIX. Pelecani earbonis.

Exemplar integrum, adauctum.

a. Proboscis apice attenuata.

b. Collum, lacre.

c. Corporis pars antica, crassissirna, subglobasa, aculeis brevibus densissime sibi adpositis armata.

d. Apex caudalis tenuis, nudus. 


\section{ECHINORHYNCHUS PORRIGENS. Balaenae rostratae.}

\section{Secundum Westrumb de helminthibus acanthocephalis (no. 16.)}

Fig. 1. Vermis integer. Magnitudo naturalis.

a. Proboscis cylindrica, receptaculo infundibiliformi insidens.

b. Reccptaculum obconicum, quo colium terminatur.

c. Collum longissimum, filiforme.

d. Corpus subaequalc, hinc illincre constrictum.

e. Apex candalis, forea praeditus.

Fig. 2. Infuudibuli apex, cum proboscide. Auctus.

a. Proboscis partim retracta.

b. Capitulum, cui proboscis, reluti raginae, insidet.

Fig. 3. Mas longitudinaliter apertus. Magnitudo naturalis,

a. Receptaculum expansum, in quo corpora globosa, in racemi formam coacerrata conspiciuntur. (De quibus ride f. 6.)

b. Collum.

c. c. c. Filum, per totan corporis longitudinem reptans, cum testiculis cohaerens:

d. Testiculus anterior.

c. Testiculus posterior.

f. Vas deferens.

g. Tesiculae seminales (in fig. 5. adauctae).

h. Fibrae musculorum longitudinalium, fascicnlos formantes.

Fig. 4. Femina longitudinaliter secta. Magnitudo naturalis:

a. Receptaculum.

b. Collum, qui nec raginam proboscidis, nec lemniscos, ut in reliquis echinorliynchis, continens, tres tantum partes offert: scilicet ligamenta tria longitudinalia arcte membranae subjacenti mucoso.reticulatae adjuncta, filum parvissimum et tenuissimum totarn cor- 
poris longitudinem legens (in maribus cum testiculis, in feminis autem cum orario et utero cohaerens) (f.), et denique in summa parte apices corporum globuliferorum (v. f. 6.)

c.c.c.c. Ovaria, duas series formantia.

d.d.d. Tria ligannenta rasculosa, totam corporis longitudinem petentia, quibus oraria inhaerent.

e. e. Fibrae musculares longitndinales.

f. Filum tenue, cum utero cohaerens.

Fig. 5. Pars postica maris, aucta.

a. Vas deferens.

b. b. Vesicae seminales.

c. c. Fibrae musculares Iongitudinales.

d.d. Fibrae musculares annulares.

Fig. 6. Receptaculum infundibuliforme, per mediam longitudinem dis. sectum. Auctum.

a. Proboscis retracta.

b. Capitulum in superficie prominens, cui proboscis continetur.

c. Corpusculum orale, cum capitulo hoc commnnicans, et usque ad mediam longitndinem receptaculi tendens. Hoc corpusculum raginae proboscidis analogon esse videtur.

d. Filum tenuissimnm in maribus cum testiculis, in feminis cum ntero colnaerens.

e. e. Duo corpora plana, plus minusre conroluta, quae a corpusculo orali (c.) ntroque in latere abcunt, forsan cum lemniscis comparanda.

f.f. Corpuscula quatuor, racemi imaginem referentia, inter musculos et corporis latus sita. Ilace corpuscula multis ex globulis constant, quorum unusquisque baccarum instar

§. ๖. §. ̊. Petiolo instructus est, et cum vase magno cohacrent, quod basin apicis plani perreptans in raginae carum hiare videtur. Dissecta naturam membranaceam et grumosam ostendebant.

Fig. 7. Pars tunicac internae feminac, aucta.

a.a. a. Ligamenta tria rasculosa, totam corporis longitudinem petentia, quae cum corporibus retortaeformibus totum corpus investicntibus (ovariis) colracrent.

b.b.b. Oraria, quorum inumquodque cum ligamento rasculoso (a.) colnacret. Onuia duas qrasi series formant, quarum quaeque utroque in latere ligamentorum illorum opé fibris annularibus musculosis subjacentibus connexa est, et inferne cum cornubus uteri (r. fig. 10. b.b.) communicat.

c. Filum tenue ab utero ad receptaculura pertinens.

Fig. S. Ovaria, cum ligamento fig. 7. a. descripto conjuncta, magis aucta. Fig. 9. Eadem sub microseopio composito, ita ut ovula immatura, oblonga vel subsphaerica, conspiciantur. 
Fig. 10. Uterus, ex corpore depromptus.

Forma linearis est, longissinus, hine illine rentricosus et constrietus.

In tria cornua finditur, quorum

a. Medium sensim sensimque in ras minutum mutatur, et per totam longitudinem currens receptaculum colli intrat.

b. Lateralia rero cum orariis, utroque in latere sitis, communicant.

Fig. 11. Ova, utero contenta.

a. Embryonis rudimentum clare conspicitur. 


\section{T A B. XIII. \\ $\begin{array}{lllllllllll}\mathbf{N} & \mathbf{E} & \mathbf{M} & \mathbf{A} & \mathbf{T} & \mathbf{O} & \mathbf{I} & \mathbf{D} & \mathbf{E} & \mathbf{A} \text {. }\end{array}$}

\section{ASCARIS LUMBRICONDES. Ex hominis intestinis.}

Secundum Jules Cloquet Anatomie des rers intestin. etc. (no. 4.)

Fig. 1. Femina, a reutre risa.

A. Os, tuberculis tribus munitum.

B. Auus.

C. Coarctatio, quae reperitur ubi anterior corporis triens cum duobus postericius trienti-. bus conjungitur.

D. Vulia, in illa coarctatione sila.

E. E. Linca abdominalis, a Viro Clarr. Cloquet pro nerro habita.

Fig. 2. Ascaris femina, a rentre longitudinaliter aperta.

A. A. Integumentum exterius, musculoso-cutaneun.

B. B. Lineae laterales.

C. Linea dorsalis (a Cloquet etiam pro nerro liabita), per appendices nutricias pellucens.

D. Oris tria tubercula a se invicem remota.

E. Oesophagus.

F.F. Tentriculus, in quo contenta, materiae lutescentes, conspiciuntur.

G. Canalis intestinalis initium, ab oraris convolutis circnmdatum.

II. Dilatatio canalis intestinalis insignis, prope suam terninationem.

1. Vagina, quae inter vasa et appendices nutricias se inserit, et exteritis aperitur.

K. Uterus, cum illa continuaus.

L. Ejusdem cornua longa, sub canali intestinali rarie flexa, ad extremitatem caudalem us. que pertinentia, in oraria transeuntia.

M.M. Ovaria conroluta, ca ratione, qua aperto abdominc plerumg̨ne inreniuntur.

N. Stratum corporis internum, per rasa et appendices nutricias formatum,

Fig. 3. Ascaris lumbricoides, mas, a laterc dextro risa.

a. Caput, et oris tria tubercula. 
b. Cauda, forma triangulari, rersus abdomen uncinatim recurra.

c. Penis, qui ex parte ani anteriore, sub forma appendicis exigui filiformis, exit.

d. Linea lateralis dextra.

Fig. 4. Eadem ascaris, rentre aperto.

a. Integnmentum cxterius.

b. b. Lineae laterales.

c. Caput.

d. Oesophagus.

e. Ventriculus.

f. Penis *), dle parte ani anteriorc exicns.

g. Vesicula seminalis, sub canali intestinali sita.

h.h. Tubus seminiferus rel testiculus, circa canalem intestinalem, quen flexionibus suis numerosis circumdat, ascendens.

Fig. 5. Os et oesoplagus ascaridis, a latere abdominis longitudinaliter aperti. Aucti.

a. Tuberculum oris superius.

b. b. 'Tubercula duo inferiora.

c. Granulationes, in interno oris reperiundae.

d. Caritas oesoplaggi triangularis.

c.e. Margines sectionis oesophagi, crassitudinem hujus organi indicantes.

f. Orificium inferius oesoplagi, forma etiam triangulari.

g. Ventriculi initium.

Fig. 6. Sectio oesophagi transtersalis. Aucta.

a. Integumentum exterins.

b. Caritas ocsophagi triangularis.

c. Fibrae carnosae, quae radiorum instar a membrana externa ad internan usque extenduntur.

Fig. 7. Portio ascaridis, ex fine anterioris corporis trientis desumta, longitudinaliter fissa, et a facic interna visa, Aucta.

a. a. a. Vasa et appendices nutriciae.

b.b.b.b. 'Terminationes rasorun nutriciorum prope lineas laterales.

c. c. Lineae laterales, in quibus ras medium conspicitur.

d. Linea abdominalis (a Cloquet pro nerro habita).

c. e. Nonnulla vasa nutricia in media parte rupta.

*) Ipse Parisiis commorans perspicue vidi, penem saltem in ea Cloqueti praeparatione, secun. dum quan liaec figura delineata est, simplicem esse, sicuti illo tempore etiam alias, ab eo factas praeparationes, in collectis MIusei historiae naturalis depositas, perlustravi. 
Hig. 8. Sectio corporis transrersalis, in medio triente anteriore facta. A u c t a.

a. Pellis.

b. Strata duo muscularia.

c. Caritas rentriculi.

d.d. Vasa absorbentia, e marginibus rentriculi nascentia, quae inter se spatium triangulare relinquunt, in quo

e.c. Lineae laterales prominent.

f. Dispositio appendicum nutriciarum circa ventriculuna.

g. Linea (nervus) dorsalis.

l. Linea (nerv us) abdominalis.

Fig. 9. Docet formam et dispositionem appendicum nutriciarum, una cum fasciculo fibrarum carnosarum longitudinalium, quibus adnexae remanebant, e corpore desumtarum.

a. Fasciculus fibrarum muscularium.

b. Appendices nutriciae.

c. Vasa nutricia partiu rupta. 


\section{T A B. XIV. \\ $\begin{array}{lllllllllll}\mathbf{N} & \mathbf{E} & \mathbf{M} & \mathbf{A} & \mathbf{T} & \mathbf{O} & \mathbf{I} & \mathbf{D} & \mathbf{E} & \mathbf{A} \text {. }\end{array}$}

\section{ASCARIS LUMBRICOIDES.}

Secundum Jules Cloquet Anatomie des vers intestinaux etc. (no. 4.)

Fig. 10. Extremitas ascaridis anterior, a rentre risa.

a. a. Tubercula oris inferiora.

b. 'Tuberculum superius.

c. Filum nerrosum abdominale, inter tubercula inferiora terminatum.

Fig. 11. Tubercula tria capitis, ab anteriori visa et a se inricem remota, ita ut excaratio eorum interna et aperturatriangularis oris con$s$ pici possit.

a. 'Iuberculum superius.

b. b. 'Tubercula inferiora.

c. c.c. Excaratio, a tuberculis interne formata.

d. Apertura oris.

e. e. Lineae punctis notatae, corporis ambitum indicantes.

Fig. 12. Unum a reliquis duobus oris separatum tuberculum, a latere $r$ is u m.

a. Sulcus, quo tuberculum a corpore separatur.

b. Facies ejus externa sire conrexa.

c. Facies ejus interna, superne angulum prominentem offerens.

d. Excaratio partis inferioris hujus faciei.

Fig. 13. Caput ascaridis, ex equo.

a. Tuberculum oris superius.

b. Strangulatio siugularis, quae ab eo versus apicem offertur.

c. Fissura, quae in ejus parte supra strangulationen sita occurrit.

d. Initium lineae (nerri) dorsalis. 


\section{- 46}

Fig. 14. Eadem portio, ab anteriore visa,
a. Tuberculum superins.
b. b. 'Tubercula isferiora.
c. Stella, formae singularis, quae per tria tubercula oris unita effingitur.

Fig. 15. Musculorum longitudinalium abdominis fibrac.

a. Extremitas anterior harum fibrarum, quae diffinduntur in duo fascicula inserta in

b. b. 'I'ubercula inferiora oris.

Fig. 16. Extremitas caudac ascaridis feminae, a latere abdominis visa.
a. Anus.
b. Punctum nigrum exignum, quod invenitur in caudae apice.
c. Finis lineae (nervi) abdominalis.
d. Corporis carum.

Fig. 17. Cauda ascaridis maris, a facic dorsali risa.
a. Eumentia hujus faciei mediana et longitudinalis.
b. Sulci, eam lateraliter cingentes.
c. Extremitas caudae.
d. Caritas corporis.

Fig. 1S. Eadem pars, a latere sinistro visa.

a. Eminentia dorsi longitudiualis.

b. Sulcus profundus hanc eminentiam lateraliter terminans.

c. Penis, de ani parte anteriori exiens.

Fig. 19. Sectio transversalis caudae maris.
a. Integamentum extcrias.
b. Caritas canalis intestinalis,
d. Vesicula seninalis.

Fig. 20. Sectio transversalis candac feminae.
a. Integumentum exterius.
b. Cavitas canalis intestinalis, integumento huic undique adhaerens.

Fig. 21. Penis Ascaridis.
a. Apex ejus poro perforatus.
b. Hijus basis, recepta in infiatione quadam, ab extremitate vesiculae seminalis et ab in- testino formata.
c. Caritas penis, pro pellucidate hujus organi visa.

Fig. 22. Portio $\Lambda$ scaridis, longitudinaliter fissa, extensa, et ita praeparata, ut diversastrata musculorum parictes caritatum splanchnicarum formallia in couspectum reniant.

a. Pellis.

b. b. Fibrae muscalares transrersales s. externae. 
c. c. Fibrae musculares tongitudinaies s. internac.

d. Appendices nutriciae, quarum bases solummodo conspiciuntur, et quae, ab hoc latere visae, parrarum resicularum pellucidarum coacervationis speciem prae se ferunt.

e. Linea (nervus) dorsalis, inter fibras musculares longitudinales et appendices nutricias descendens.

f. Linea lateralis dextra.

g. Facies abdominalis corporis.

Fig. 23. Extremitas anterior Ascaridis ex equo, in longitudinem lateris abdominis fissa, ut modum anionis linearum lateralium doceat.

a. Duo tubercula inferiora oris.

b. Oesophagus.

c. Ventriculi initium.

d. d. Lineae laterales.

e. Arcus anastomoticus, quem duo rasa mediana linearum latcralium sub oesophago inter se formant.

f. Unio anbeduarum linearun lateralium circa oris tubercula et oesophagi initium. 


\section{T A B. XV. \\ $\begin{array}{lllllllllll}\mathbf{N} & \mathbf{E} & \mathbf{M} & \mathbf{A} & \mathbf{T} & \mathbf{O} & \mathbf{I} & \mathbf{D} & \mathbf{E} & \mathbf{A} \text {. }\end{array}$}

\section{ASCARIS LUMBRICOIDES.}

Secundum Jules Cloquet (no. 4.)

Fig. 24. Organa genitalia $\Lambda$ scaridis feminae.
a. Vagina.
b. Uterus.
c. Uteri cornna.
d. Flexiones ovarii expeditae et ab invicem separatac.
e. Extremitas ovarii ultima.

Fig, 25. Organa generationis Ascaridis maris, ex corpore desumta.

a. Penis.

b. Vesicula seminalis.

c. Tubus seminiferus s, testiculus.

d. Tubi scminiferi finis, nodo parro formatns, qui flocci grisei vasis nutriciis affixi speciem praebet.

Fig. 26. Materia grumosa, albicans, cujus moleculac irregulares, et amorphae sunt, quae in ultimis ovariorum ramificationibus reperitur.

Fig. 27. Materia alba, ovariis media eor um longitudine inclusa. Ea corpusculis lincaribus, acuminatis in una et obtusis in altera earum extremitate, formata est.

Fig. 28. Ora, per commorationem in spirito rini, deformia.

Fig. 29. Ora in ovariis, distantia quadam a loco ubi cum cornubus uteri continuantur, contenta. 
Fig. 30. Ora, ex cornum nteri extremitate et ex initio ovariorum desum ta.

Fig. 31. Ora triangularia, uterum Ascaridis replentia.

Fig. 32. Ora subrotunda, nunc regularia, nunc irregularia et quodammodo gibbosa, in magno Ascaridum numero reperiunda.

Fig. 33. Ova, ex utcro prope vaginam desumta, perfecte inatura.

Fig. 34. Ora per commorationem in spirito vini deformia.

Fig. 35. Liquor in resicula scminali maris inclusus.

Fig. 36. Liquor in ramificationibus canalis spermatici contentus. 


\section{T A B. XVI.}

\section{$\begin{array}{lllllllllll}\mathbf{N} & \mathbf{E} & \mathbf{M} & \mathbf{A} & \mathbf{T} & \mathbf{0} & \mathbf{I} & \mathbf{D} & \mathbf{E} & \mathbf{A} \text {. }\end{array}$}

Secundum Bojanus Enthelminthica (no. 1.)

FIG. 37. - 48. ASCARIS LUNBRICOIDES.

Fig. 37. Musculorum longitudinalium fasciculi quatuor extensi, magnitudiue naturali.

i. i.i. Spatia inter fibras concurrentes.

Fig. 38. Fibrarum muscularium circularium, externe sitarum, portio extensa et admodum aucta. Varia anastomosis inter fibras antcrius et postcrius sitas in couspectum renit.

Fig. 39. Portio corporis longitudinaliter juxta lineas laterales fissa ct extensa, ut resicularum series conspiciantur, multum aucta.

c. c. Linea utraque lateralis.

n. n. o. o. Quatuor series vesicularum.

p.p.p. Corpora vesicularum.

q.q.q.q. Crura resicularum, in situ naturali canali intestinali adhaerentia, hic rero libera. Altcra harum resicularum crura lineae dorsali aut abdominali adhuc affixa sunt.

s. Linea dorsalis.

ss. Linea abdominalis.

Fig. 40. Vesiculae aliquot, cum linea dorsali conjunctae, multum auctae. Lineae abdominali adhaerentes resiculae eadem forma ga u dent.

s. s. Portio lineae dorsalis.

p. Corpora vesicularum, rotundata et cucullata, 
q. เ. Crura resicularum; scilicet

q.q.q. Crura jam libera, a canali intestinali rel a musculo longitudinali solnta.

r.r.r. r. Crura lineae dorsali inhaerentia.

Fig. 41. Sectio transversalis Ascaridis, multum a cta. Cavitati corporis inspicitur et vesiculac in icone clarius, quam in natura apparent, cxpressae sunt, ut melius illustrentur.

a. a. Fibrac muscularum circularium.

b. b. Musculi quatuor longitudiuales, transtersim secti.

c. c. Linea utraque lateralis.

s. Linea dorsalis.

ss. Linca abdominalis. Hae duae interue minus quam lincac laterales prominent, et vix a musculis longitudinalibus recedunt.

n.n. o. o. Quatuor resiculannm scries.

p.p.p. Corpora resicularum.

q.q. Crura resicularum cauali intestinali adhaerentia.

qๆ. Crura resicularum musculis longitudinalibus affixa.

r. Crus quoddam lineac dorsali adhaerens.

t. Paries canalis intestinalis, transrersim secta.

u. Carum canalis intestinalis.

r.r.w.w. Quatuor spatia inter parietem canalis intestinalis et parietem corporis, in quibus resiculae reperiuntur. Ea quae lineis lateralibus proxima sunt (v. v.) canalem longitudinalem, inter lineas laterales, crura resicularum et parietes intestini situm, formant.

Fig. 42. 43. 41. 45. Vesiculac separatae.

42. 43. liotundatae,

44. 45. Cucullatac.
p. Corpora,
q. r. Crura resicularum.

Fig. 46. Ascaris lumbricoidcs, mas, cx equo, a latere visa. Magnitudo uaturalis.

a. 'Tubercula tria capitis.

b. Genitale masculum, paullulum ante cxtremitatem caudalem spicula duplici prominens.

c. Lineac laterales.

d. e. Corpora fasciculata quaedam, in latere sinistro pellucentia.

f. Vasa scminifera translucentia. 


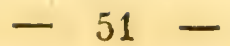

Fig. 47. Portio lineae lateralis, multum auctac.

k. Canalis hujus lineac medianus, sinuatus.

1.1. Vagina parencliymatosa eum circumdans.

m. Corpus fasciculatum (fig. 46. d.e.), lineae adhaerens.

FIG. 48. ASCARIS $\Lambda$ CUS. Ex intestino Esocis lucii.

Linea lateralis, rermis viri, multum adaucta.

k.k. Canalis, hujus lineae medianus.

1.1. Vagina parenchymatosa linjus canalis.

$x . x$ Pori s. stigmata in canali mediano obrenientia. 


\section{T A B. XVII.

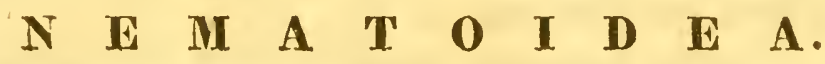

Fig. 1. - 4. secundum Goeze Naturgeschichte der Eingerveidewürmer (no. 6.) - Fig. 5. - 16. secundum Nitzsch in Ersch und Gruber Encyclopaidie (no. 10.)

FIG. 1. - 4. ASCARIS VERMICULARIS. Ex hominis intestinis crassis.

Fig. 1. Femina, magnitudine naturali.

Fig. 2. Eadem, ad a ucta.

a. Caput membrana utrinque resiculari alatum.

b. Oesopliagus.

c. Yentriculus.

d. Vagina.

e. Ora.

f. Finis tractus intestinalis.

s. Anus.

1. Cauda recta, subulata.

Fig. 3. Femina oris non stipata, a Goezio pro mare habita.

a.b.c. Denolant idem, quod in fig. praecedente.

e.f. Tractus intestinalis, ano terminatus.

Fig. 4. Ora orato-elliptica, parte media nigro-irrorata.

FIG. 5. -. ASCARIS (IIEDRURIS) ANDROPIIORA. Ex rentriculo T'ritonis taeniati. MAGNITUDINE AUCTA.

Fig, 5. Femina acetabuli, in cauda extrema reperiundi, ope parieti ventriculi interno affixa est, eique mas, spiraliter eamamplectens, inhacret.

Mas uon coitus tautum causa feminam amplectitur, sed acetabulo suctorio in cauda extrema prisatus, per feminam etiam lac ratione in rentriculo retinetur, et ab expulsione per motum intestinorum peristalticum defenditur. Nonunqquain etiam duo mares uni feminae insidentes a Vir. Clarr. Nitzsch reperti sunt. 

a. Caput feminac.
b. Caput maris.
c. Extremitas caudalis feminae, acetabuli suctorii ope rentricnlo insilens.
d. Extrcmilas caudalis maris, acuta.
c. e. Tractus intestinalis aequalis, in utroque rerme per corpus translucens.

Fig. 6. Pars postica maris.
a. Anus.
b. Tractus intestinalis translucens.
c. Papillae, ad maren sustinendum destinatae, quibus femina infima parte gaudet.
d. Apex caudalis acutus.

Fig. 7. Fluidum in orario eontentum.
a. Cotyledoncs, irregulariter rotundati, opaci, mixti inter
b. Ora elliptica, pellucida.

FIG. 8. et 9. ASCARIS OXYURA. Nitzsch. Ex intestinis crassis Muris musculi. AUCTA.

Fig. 8. Femina gravida.
a. Vulva, in parte corporis anteriore.
b. Anus.
c. Caput alatum.
d. Oesophagus amplitudinc subaequalis:
e. Ventriculus globosus.
- f. Intestini initium rotundatum.
g. Intestini finis tenuior.
l. Ora, in interuo contenta.
i. Apex caudalis.

Fig. 9. Ora magna lanceolata so fusiformia.

FIG. 10. - 12. ASCARIS TETRAP'IERA. Nitzsch. AUCTA. Ex intestinis crassis Muris musculi.

Fig. 10. Mas, a latere dextro risns:

$$
\text { Intestina per corpus translucent. }
$$
A. Caput, membrana singulari, postice acute recisa, alatum.
B. Corpus subaequale, postice non attenuatum.
C. Extremitas caudalis currata et brevis.
a. Anus, spiculis parum prominentibus.
b. Oesoplagus, aequali crassitudine.
c. Ventriculus globosus, parrus.
d.d. Tractus intestinalis aequali diametro ad caudam usque procedens.
e. Genitalia.


Fig. 11. Femina, a latere dextro.
A. Caput alatum.
B. Corpus postice vix tenuior.
C. Extremitas caudalis longa et recta.
a. Anus.
b. Oesoplagus.
c. Ventriculus.
d. Tractus intestinalis.
e. e. Orarium ovis repletum.

Fig. 12. Ora oblonga.

FIG. 13. - 16. ASCARIS SEMITEleS. Ex Charadrii Fanelli intestinis. AUCTA

Fig. 13. Feminae corporis pars.
a. Vulra.
b. Oriductus.

Fig. 14. Feminae pars postica.

a. Anus.

b. Tractus intestinalis.

Fig. 15. Maris pars postica.

a. Spicula exserta.

b. 'Tractus intestinalis.

Fig. 16. Ora. 


\section{T A B. XVIII.}

\section{$\begin{array}{lllllllllll}\mathbf{N} & \mathbf{E} & \mathbf{M} & \mathbf{A} & \mathbf{T} & \mathbf{O} & \mathbf{I} & \mathbf{D} & \mathbf{E} & \mathbf{A} \text {. }\end{array}$}

Fig. 1. - 6. secundum Nitzsch in Ersch u. Gruber Encycl. (no. 10.). - Hig. \%. - 9. secundum Bremser Lebende Würmer im lebenden Menschen (no. 2.). - Fig. 10. - 15. secundum Westrumb Anatom. d. Strongylus Gigas (no. 16.). - Fig. 16. secundum Olfers Com. de veget. et anim. corpor. etc. (no. 22.).

FIG, 1. 6. ASCARIS REFLEXA. AUCTA. Ex Caprimulgi europaei intestinis crassis.

Fig. 1. Pars ejus antica.

a. Caput distincte triralve.

b. Membrana alaris, antice rotundata, postice acuta et sensim diffluens.

c. Oesophagus longus.

d. Ventriculus globosus et parvus.

e. Intestini initium subglobosum.

Fig. 2. Maris pars media.

a. Tractus intestinalis subjacens.

b. Vas seminiferum alternis flexibus instructum.

Fig. 3. Feminae pars media.

a. 'Tractus intcstinalis subjacens,

b. Ova.

Fig. 4. Feminae pars postica, extremitate caudali longa, recta, attenuata, acuminata.
a. Anus.
b. Intestini pars ultima.
c. Ora.
d. Acumen caudale brere, subtile, distinctum.

Fig. 5. Maris pars postica.

Extremitas caudalis femina brevior, incurvata, membranae angustae ope alata, et papillis quinque instructa est. 
a. Spicula exserta, longissima.

b. Papillae quinque plano inferiori insidentes, quarum prima ante anum et ostiam gsnitale nuitum (d.) situm est, ad mas in coitu sustinendum destinatae.

Fig. 6. Ova, rotuludo-elliptica.

a. Immatura.

b. Matura, quibus cmbryo spiralis inest.

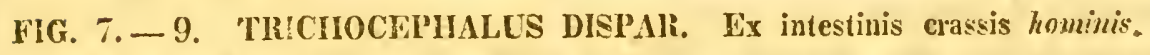

Fig. 7. Nas magnitudine naturali, parte postica spiraliter contorta

Fig. 8. Femina magnitudine naturali, parte postica rectinscula.

Fig. 9. Mas, multum adauctus.

Tractus intestinalis et rasa seminifera inflexa per corpus trausluceut.

a. Genitale masculum, ragina pelluciủa inclusum.

FIG. 10. - 15. S'IzONGYLUS ARMATUS. Ex intestinis Fifqui et Asimi,

Fig. 1a. Femina longitudinaliter dissecta. Magnitudine aucta.

A. Caput glohosum, antice obtusum, margine crasse, in quo aculei parvi, recti, denet sibi appositi reneriuntur, postice per

B. Stricturam a corpore tenuiare separatum.

C. Corpus ad caudam attenatum.

a. Canalis intestinalis, a capite usque ad candam subaequali crassitudine decurrems, in cauda

z. Ano se finiens.

b. Linea lateralis.

c. c. Ovaria duo oblonga, ad atramque canalis intestinalis latus sita, ovis repleta.

d. Vagina, primo cyliudrica, dein dilatationem quandam formans, ex qua oriuntur

g.g. Duo rasa brevia, cum orariis cohaerentia.

e. e. Vasa subtilia, ex utroque ovario ortum ducentia, quae rarie flexa ad caput usque procedunt, ibique eranescunt.

Fig. 11. Mas dissectas. Eadem ratione qua femina a ctus.

A. Caput.

B. Strictura, qua hoc a corpore separator.

D. Bursa.

a. Canalis intestinalis.

z. Anus, in mare etiam ad emittendam penem destinatus.

b. Linea lateralis.

f. f. Uterque testiculus per vas quoddam coliaerens.

g. Vas seminiferum, quod superne circa eanalem intestinalem evanescere vidctor.

b. Vas deferens, primo rectiusculum, dein rarie flexum, lincam circiter ab extremitate caudali remotum nodulum formans, ex quo prodit

i. Penis, tanquam filum corneum, subtile. 


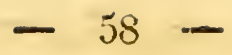

Fig. 12. Extrema cauda maris adaucta.

a. Penis, prodiens ex

b. Bursa, membrana tenui radiata efficta, in tres rel plures lobulos fissa.

Fig. 13. Portio membranac internae, adaucta.

a. Lineac laterales, utrinque canalis intestinalis sitae, prominentes. Ilae sub microscopio visae Janc structuram ostendunt. Medio harum linearum

x. Vas subtile decurrit, ad cujus iatera

у. Corpora obscura, cara, retortis s. resiculis similia sila sunt, quae inter se per illur vas cohaerent.

b. Vas ramosun, in medio corpore sub intestino situm, eaque solummodo remoto con. spicuum. Ejus rami cum corporibus vesicnlosis (y.) conjuncti esse videntur.

c. Fibrac musculares longitudinales, ab invicem remotae.

Fig. 14. Termes in coitu. Magnitudo naturalis.

a. Femina, iı gุua vulva sex lineas circiter ab extremitate caudali sub forma macular: brunac reperitur.

b. Mas, femina constanter brevior, bursa corpus ejus amplectens.

Fig. 15. Ora, embryone instructa.

FIG. 16. STRONGYLUS ELEGANS.

Orula.

a. Pars media obscurior.

b. Mlargo pellncidus.

c. Appendices, per quas orario inhaerent. 


\section{T A B. XIX.}

\section{$\begin{array}{lllllllllll}\mathbf{N} & \mathbf{E} & \mathbf{M} & \mathbf{A} & \mathbf{T} & \mathbf{O} & \mathbf{I} & \mathbf{D} & \mathbf{E} & \mathbf{A} \text {. }\end{array}$}

\section{S'TRONGYLUS GIGAS.}

Fig. 1. 6. \%. secundum Rudolphi Entoz. Synops. (no. 14.) (ex phoca desumtus). - Fig. 2. - 5. secundum Otto Nerrensystem der Eingerveidewïrmer. (no. 12.) (ex lupa).

Fig. 1. Vermis maguitudine naturali.
a. Caput.
b. Bursa.
c. Genitale masculum.

Fig. 2. Cutis feminae, ab interno risa.

A. Extremitas anterior.

B. Extremitas caudalis.

a.b.c. Filum nerreum, in sulco mediano lateris abdominalis rermis uudis oculis conspichum. Id in rerme recenti colore albo nitido, crassiticn pili adacquans, reperitur. Sub initio ocsophagi a ganglio majori oblongo (a.) originem ducit. 'T'unc nudis oculis crassitie subaequali, sed sub lente perspicue nodulostum, procedit, ex quibus nodulis (s. parris gangliis) fila tenuissima exoriuntur, quae utrinque in cutem transcunt. Denique sub finc tractus intestinalis ganglio oblongo (c.) terminatur.

a. Gauglion anterius maguum.

b. Locus ubi nerrus (in femina) sinistrorsum incurratur et arcum parrum formans raginam circumit.

c. Ganglion posterits magnum.

d. Fibrac musculares annulares.

e. e. Fibrae musculares longitudinales. Ifarum fibrarum octo sunt, quae a capite inde usque ad caudam non interruptac procedunt, et

f. f. Sulcis profundis a se invicem scparantur.

s. s. Corpuscula rotunda, mollia, semipellucida, quibus snperficies cutis interna obdacitur. In medio corpore milii granum magnitudine adaequant, in utraque extremitate rero 
sensim minuuntur. Haec corpuscula in octo series longitudinales ita disposita sunt, it totidem striis muscularibus longitudinalibus insideant.

Fig. 3. Femina dissecta. Intestina situ param mutata sunt. Fibrae musculorum circulares ac longitudinales, et inter eas sulci, nec minus corpuscula rotunda in conspecturn veniunt.
A. Extremitas anterior.
B. Extremitas caudalis.
a. Oesophagus,
b. b. Tractus intestinorum.
c. Vulva,
cc. Vagina.
d.d.d.d. Uterus, initio crassus, tunc tennis huc et illuc flexus, demum iterum crassus.
c. Dilatatio ejus (s. saccus coccus) prope extremitatem caudalem.
f. Transitus ejusdem in tractum intestinalem.
g. Ganglion anterius nervi.
h. $h$, Nervus in medio corpore decurrens.
i. i. Corpuscula rotunda.

Fig. 4. Mas dissectus.

Intestina situ fere naturali. Tractus intestinalis, musculi et corpuscula ut supra.

A. B. Extremitas atraque.

a. a. Genitale masculum ad latus intestini dorsale procerieus, demum evanescens.

b. Initium utriusqne rasis seminiferi, semine albo repleti.

bb. bb. Ejus contortiones.

c. Finis unius in bursam.

d. Finis alterius in tractum intestinalem.

Fig. 5. Initium vasorum seminiferorum in mare altero.

Fig. 6. Caput lentis ope visum, cum osculo medio et sex papillis, idem ambientibus.

Fig. 7. Cauda maris extrema, adaucta.

a. Pars discissa.

b. Bursae cavum.

c. Filum s. genitale masculum, ex papilla oriundan. 


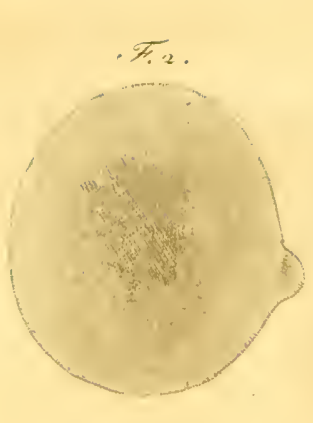

$$
\text { ryestrier. }
$$

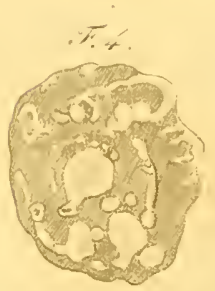

.4 .5$.
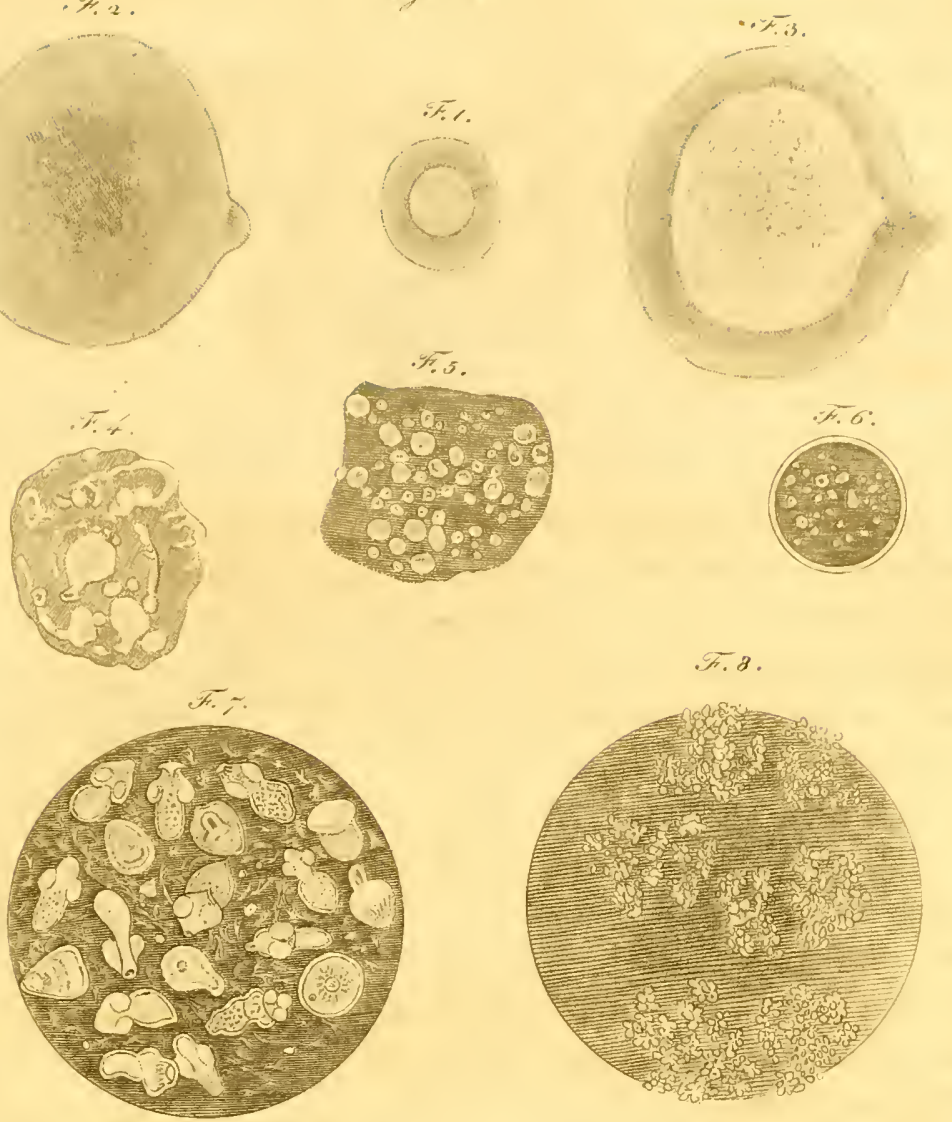

$F_{12}$. .5. 10. 9.

a (6) (C) bf (0)

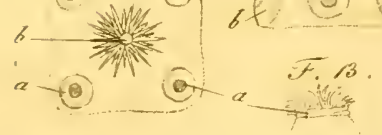

$$
6-3
$$

$\sum_{a}^{2} 09$.

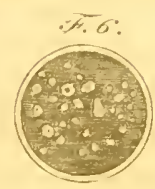

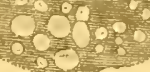
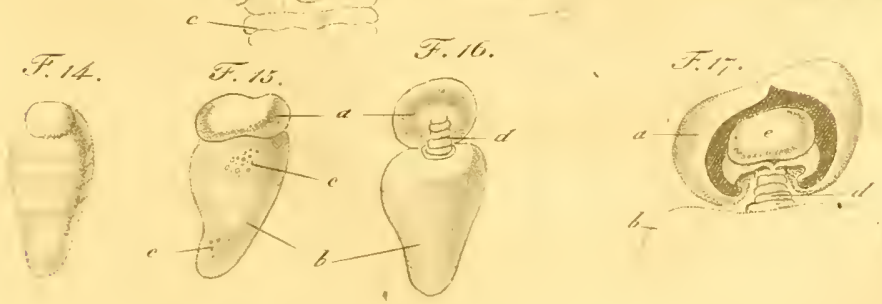

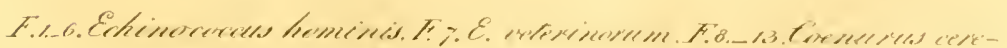

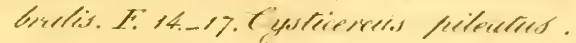





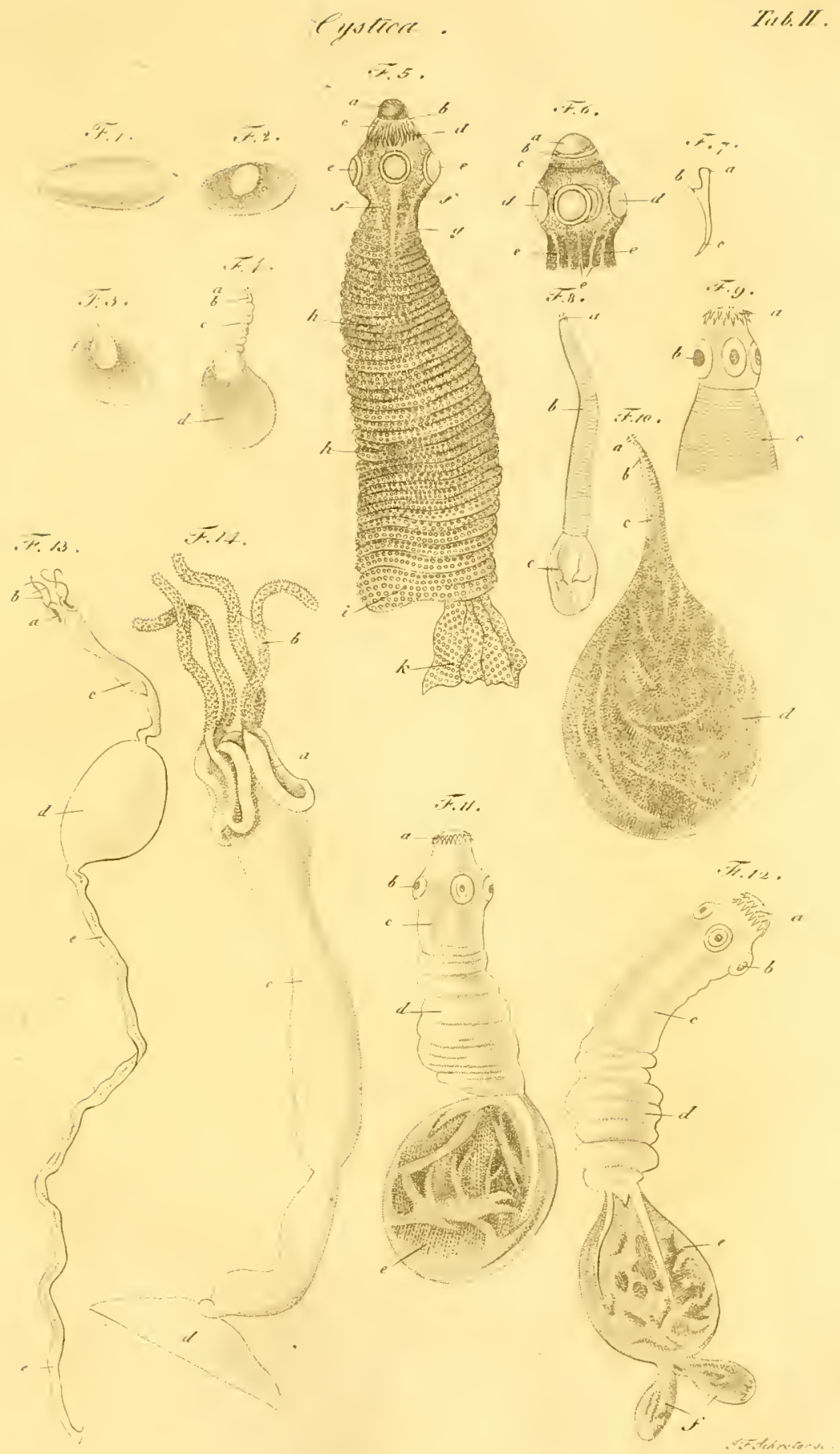

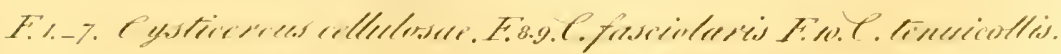

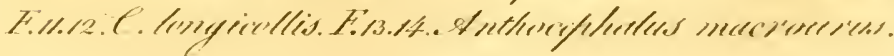




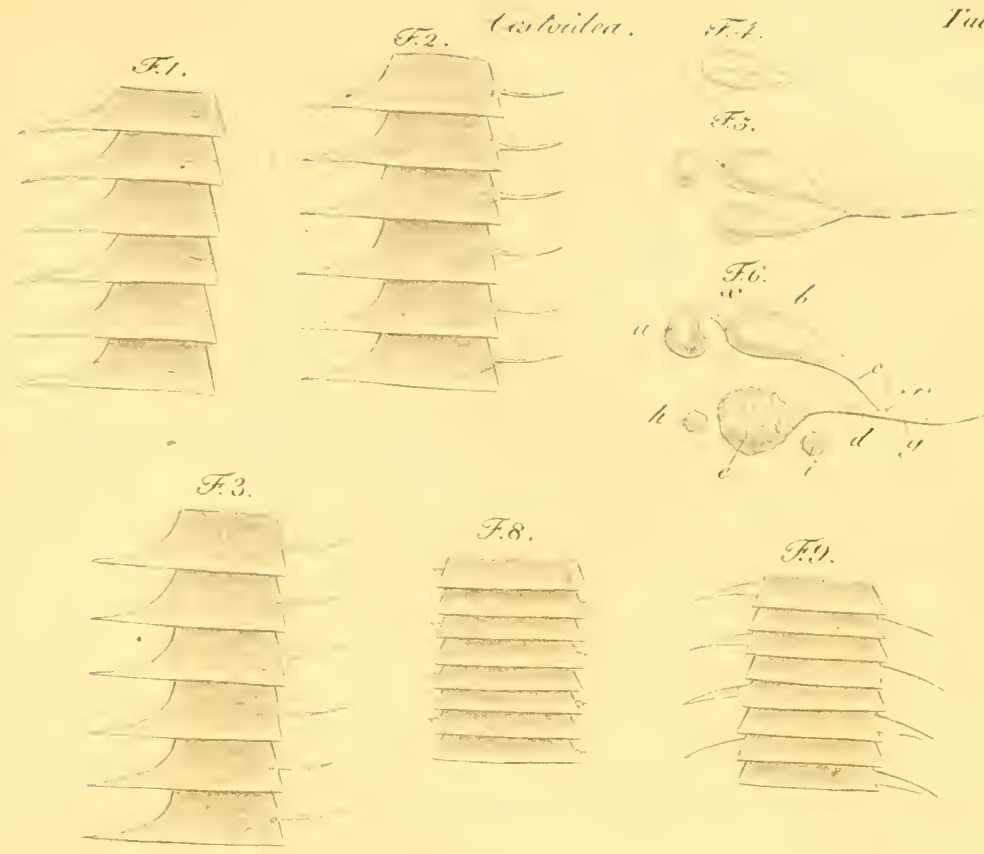

FIe.
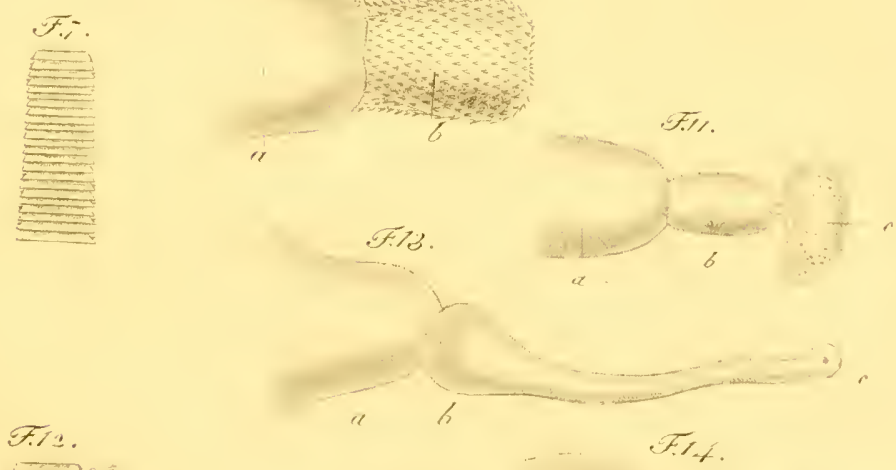

FY.

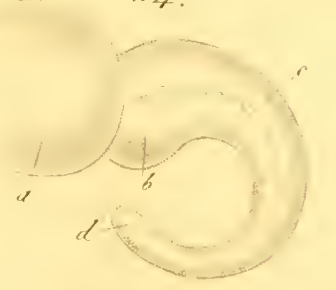



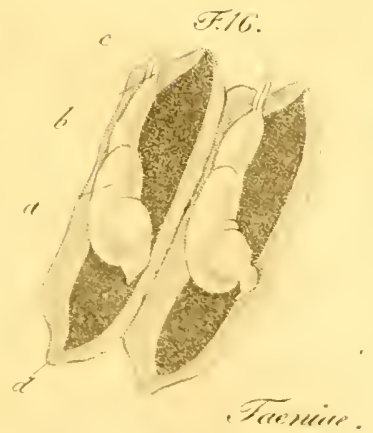

- 51.5.

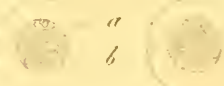

- Treverier 



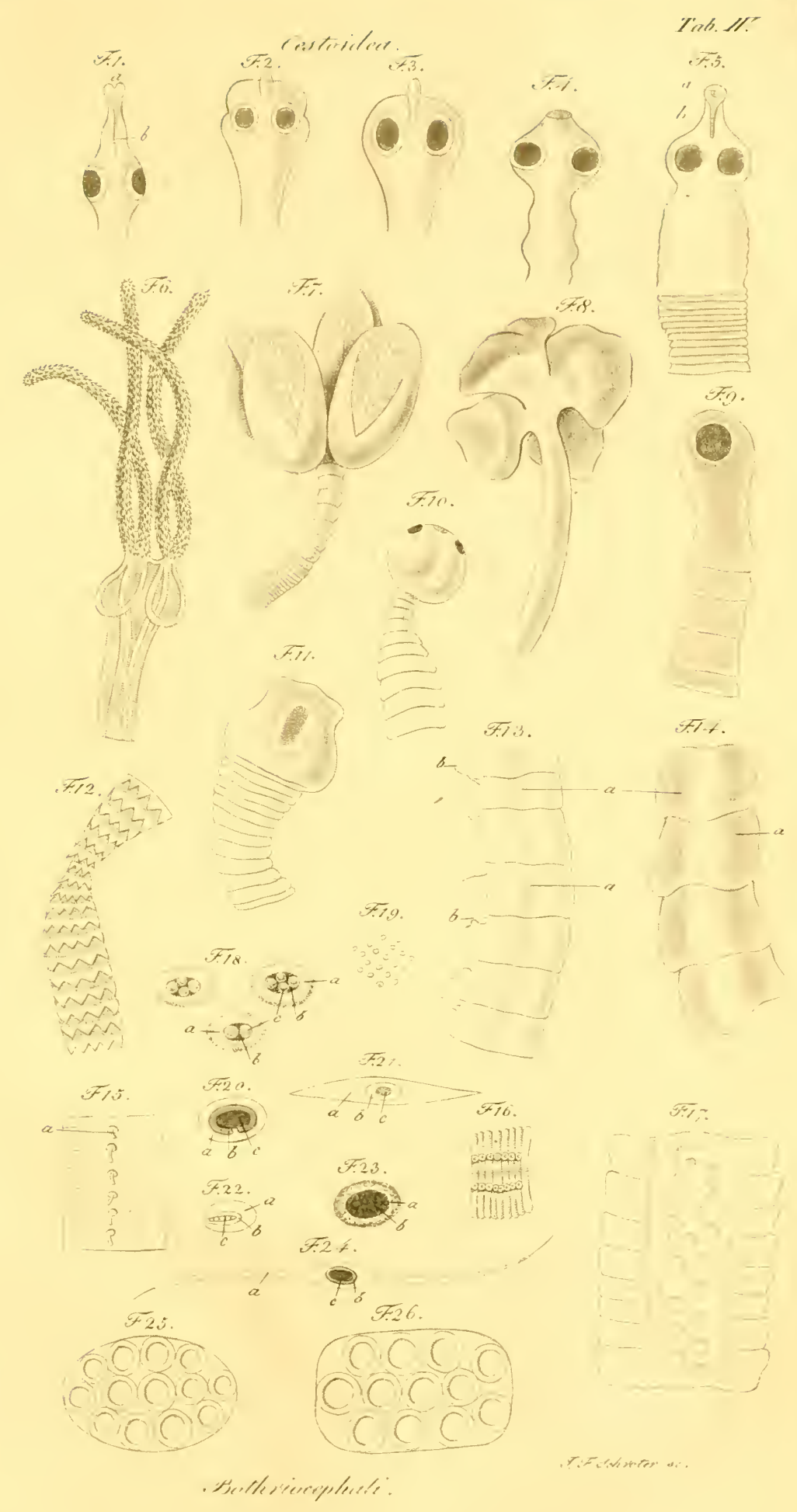





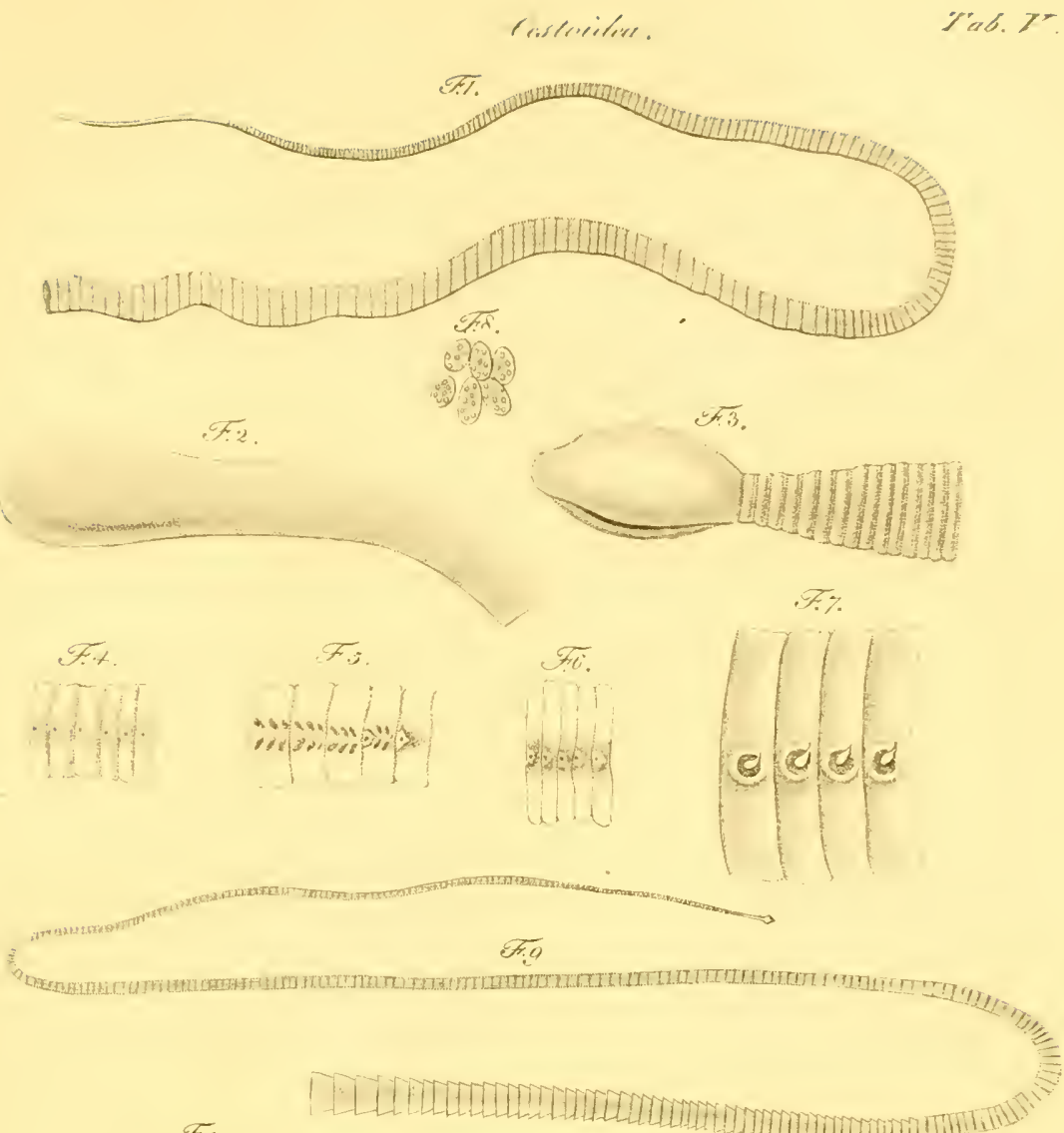

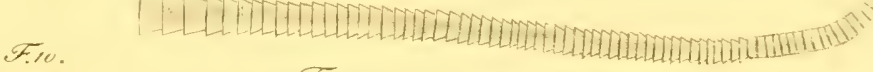

$\therefore$

(a) 6

F.13.

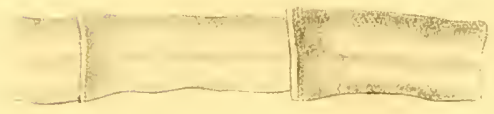

F. T.

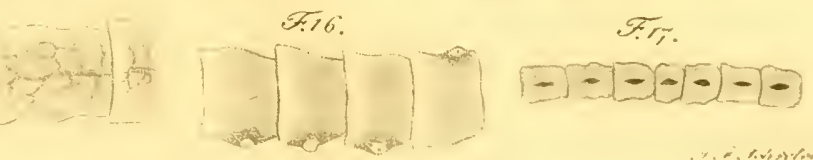

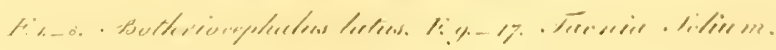

कार.
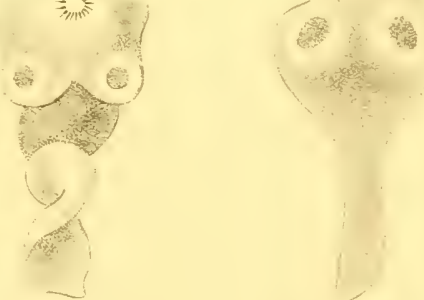

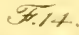

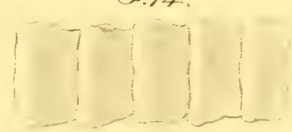

.... 


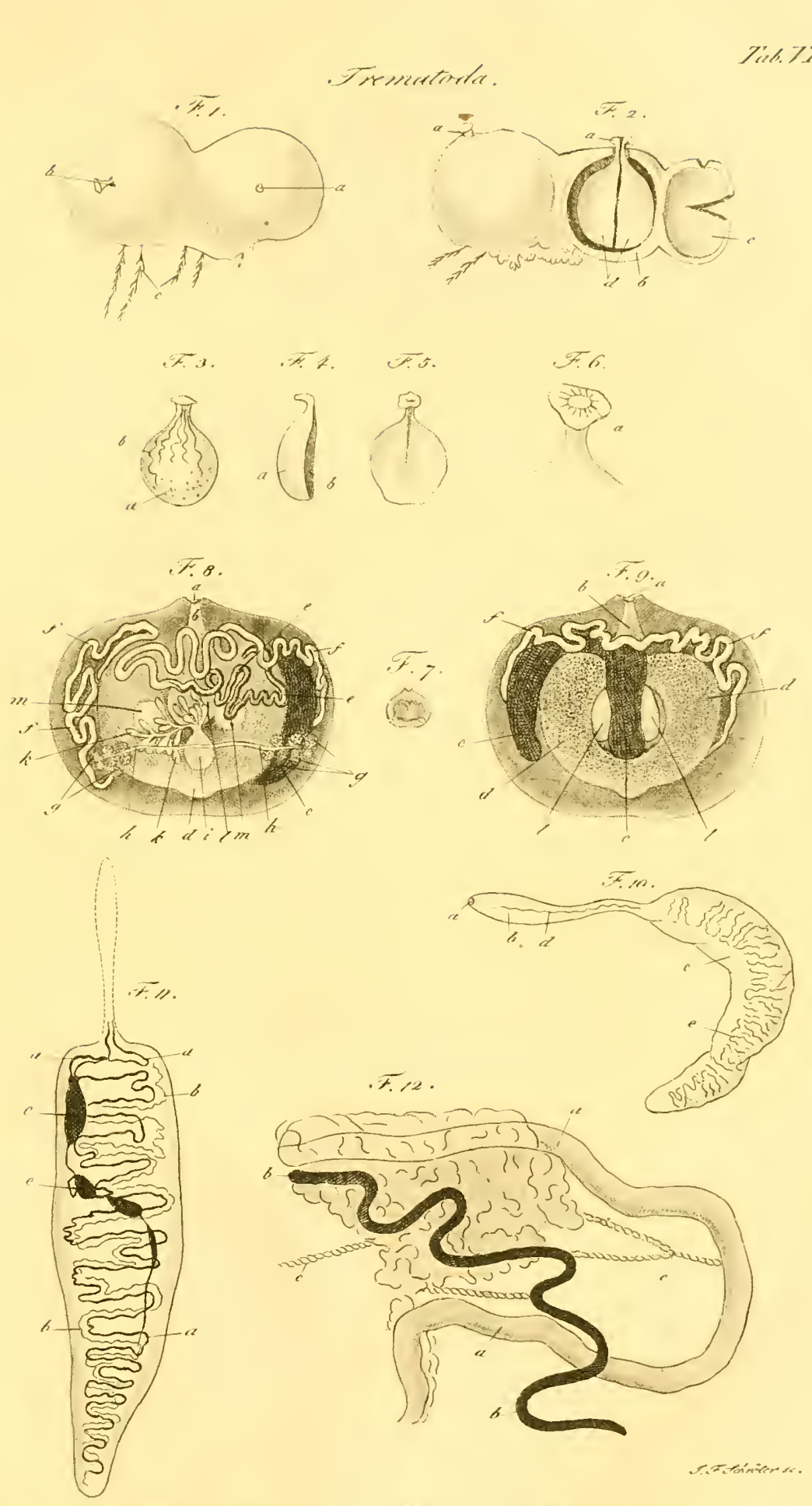

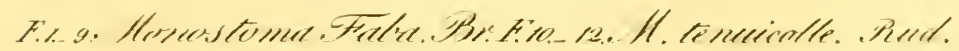





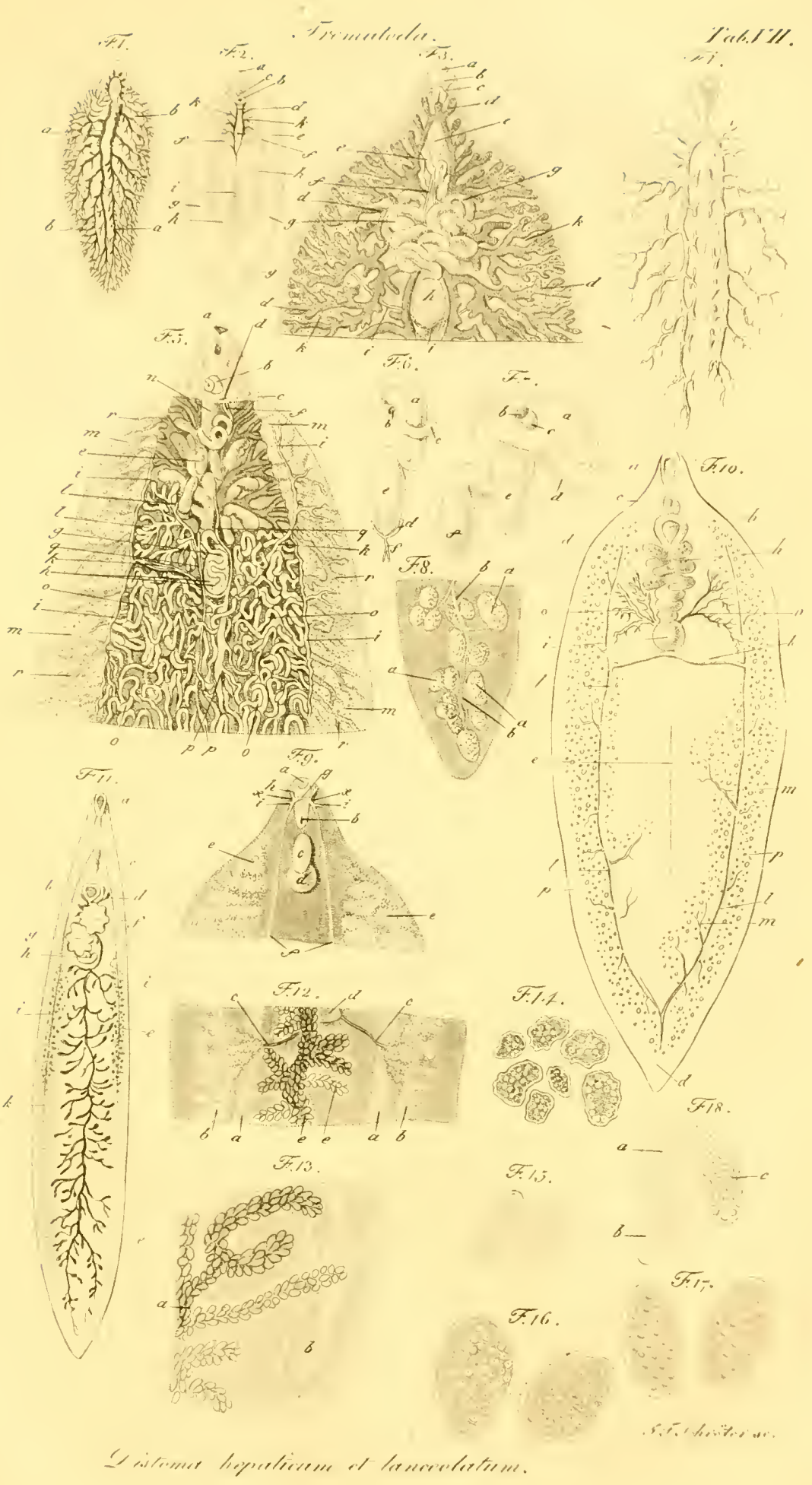




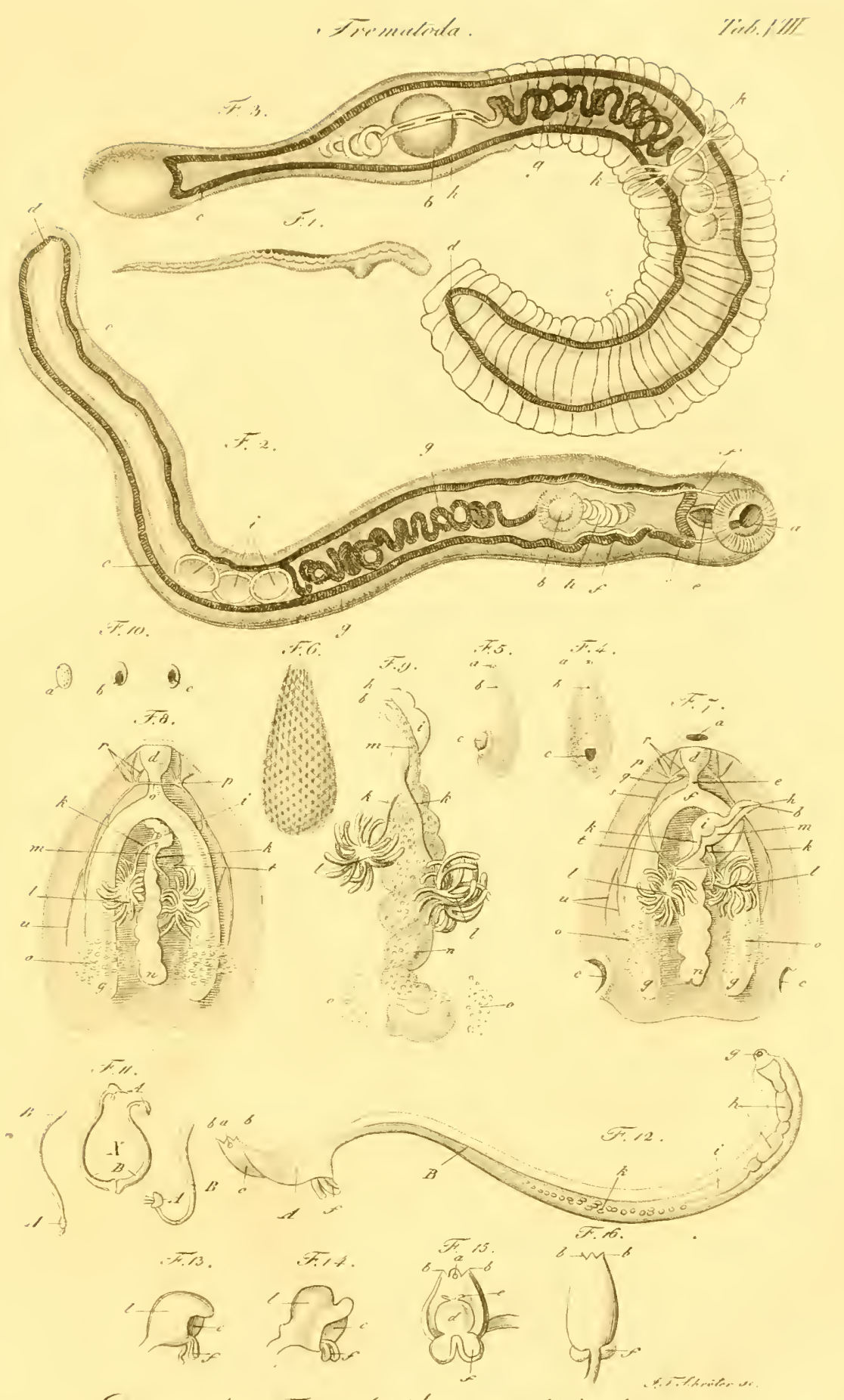

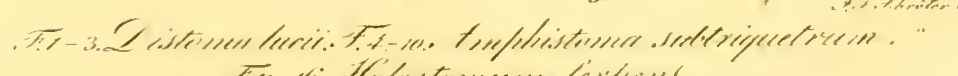

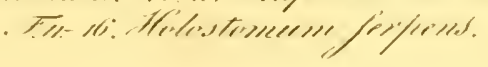





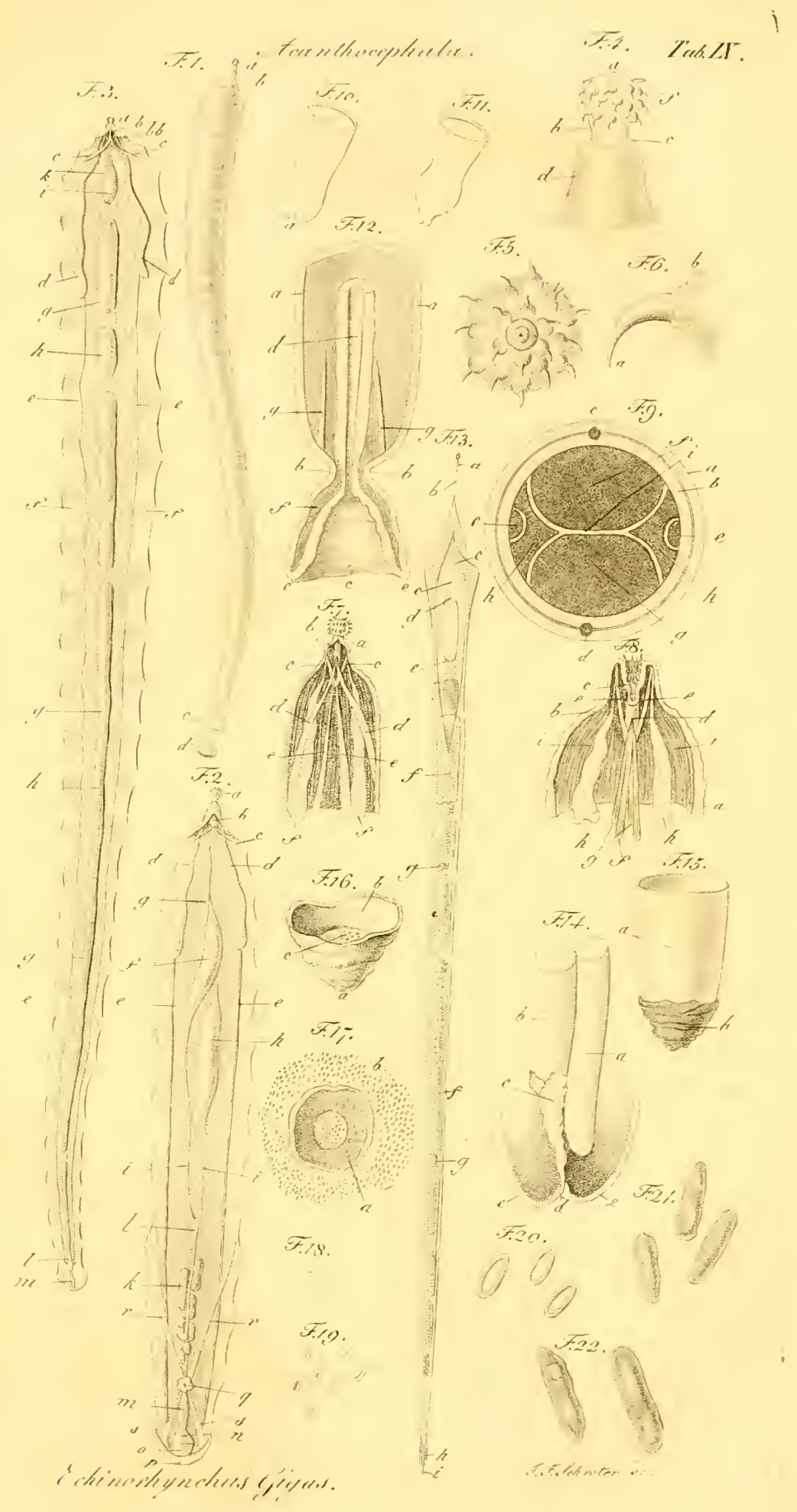





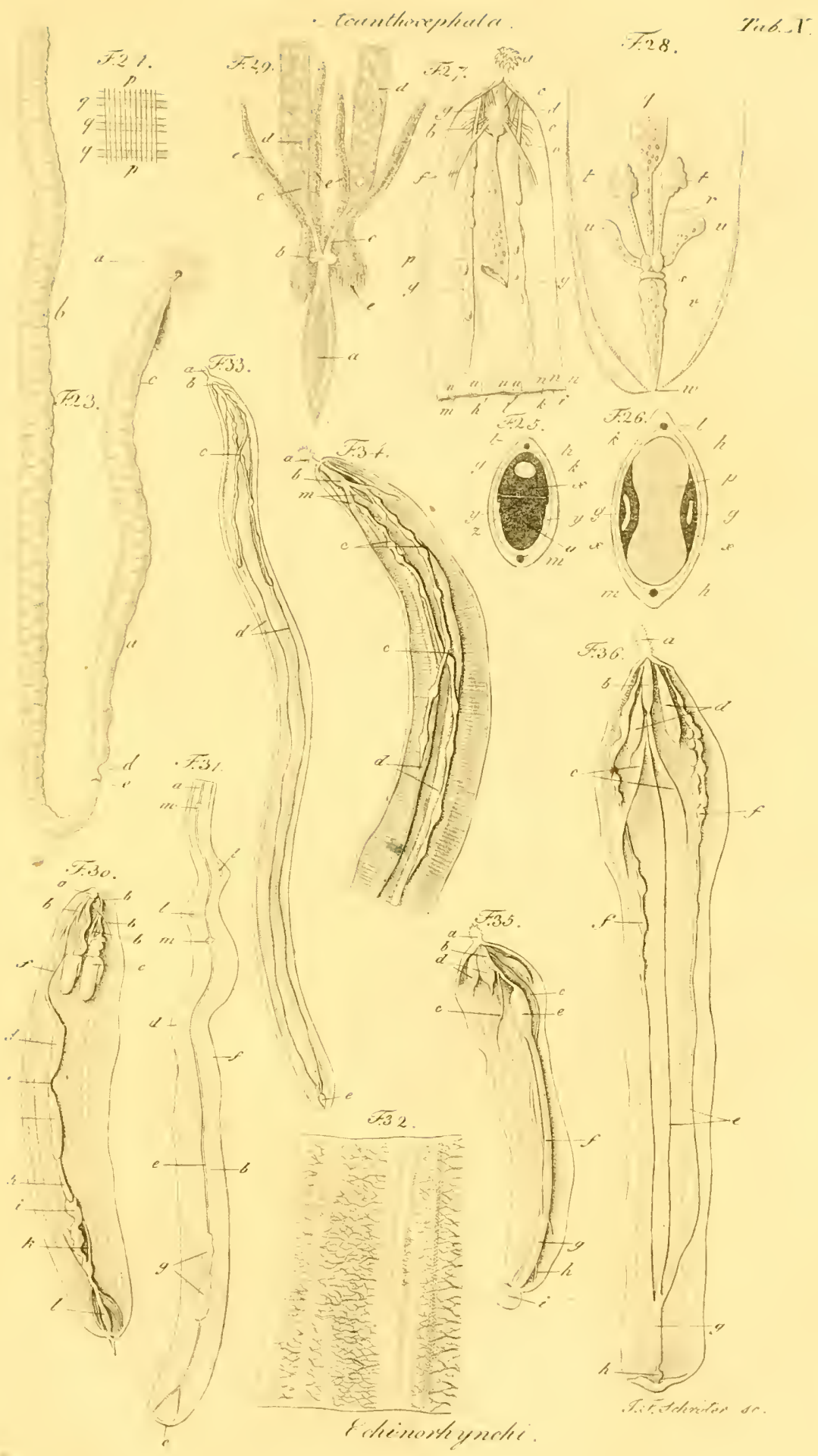





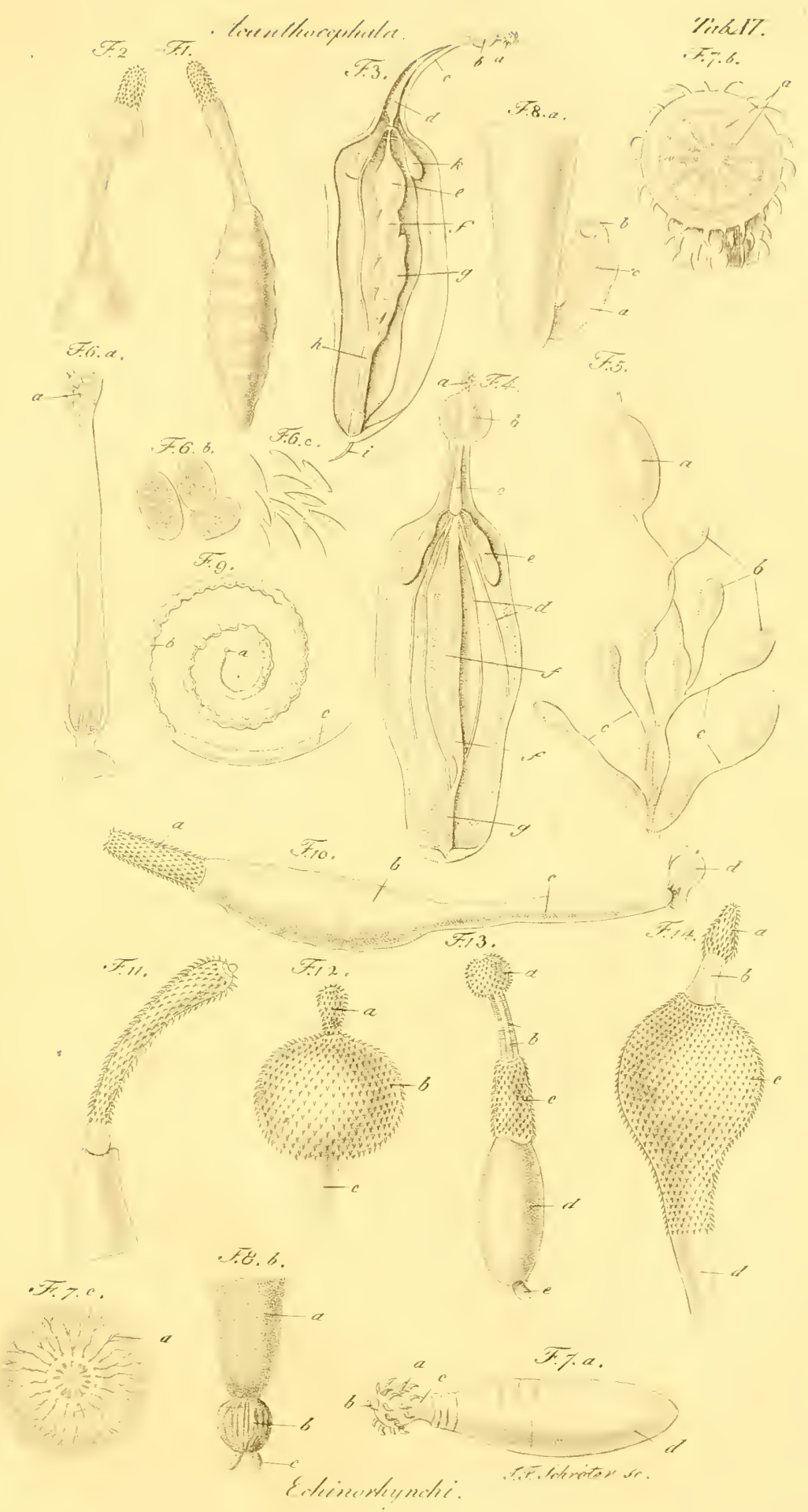





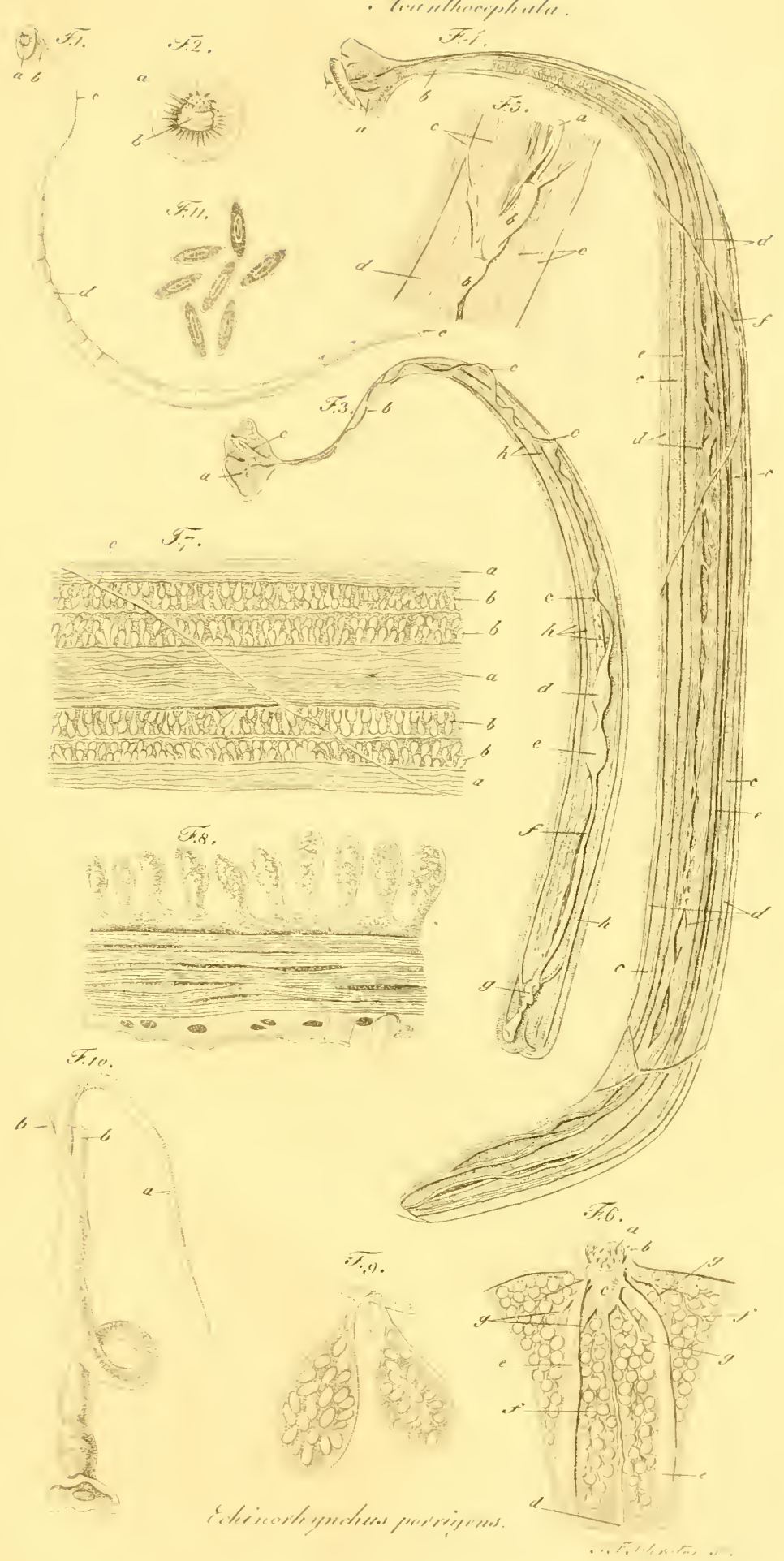





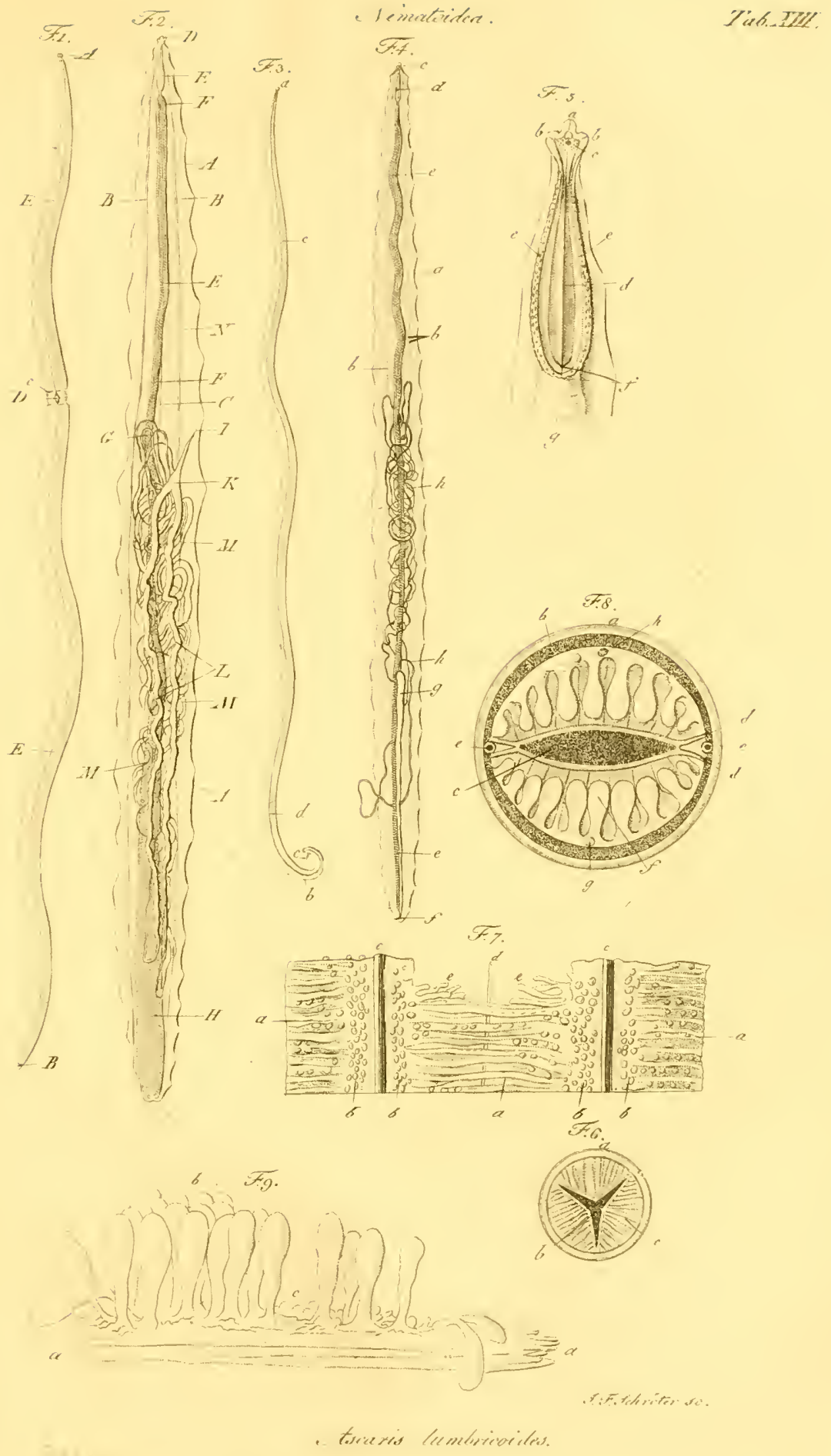




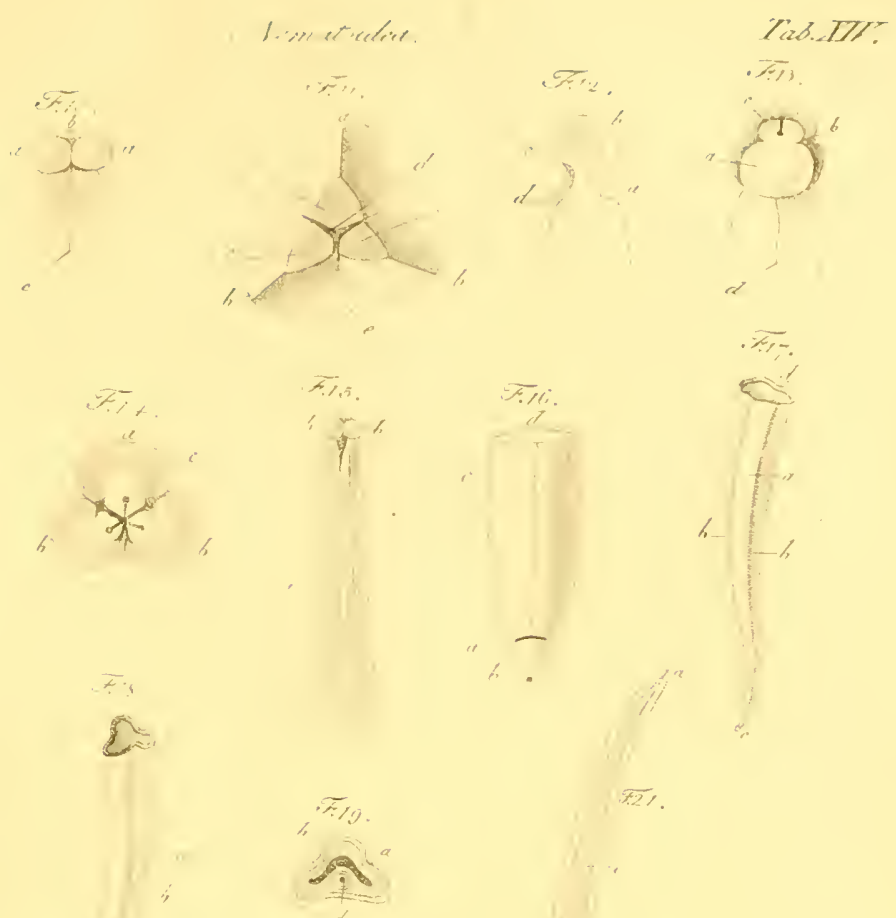

(F).
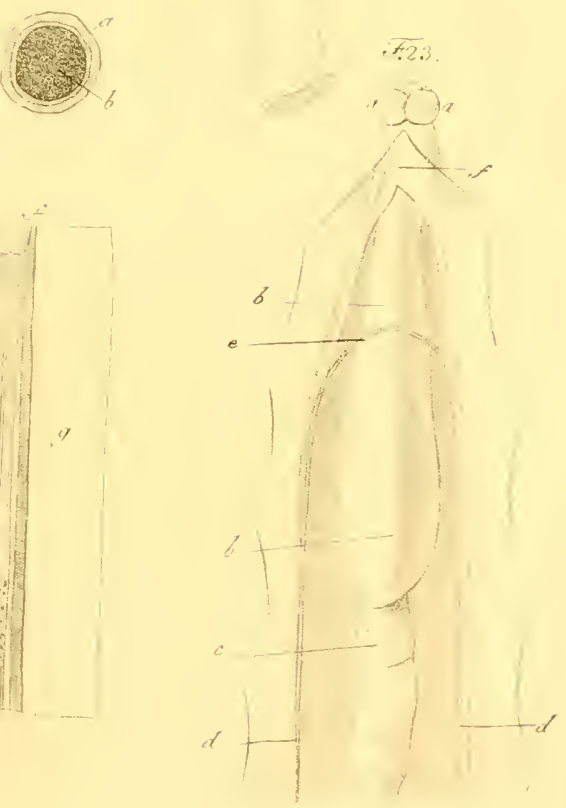

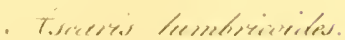

. T.T.Trencter de. 



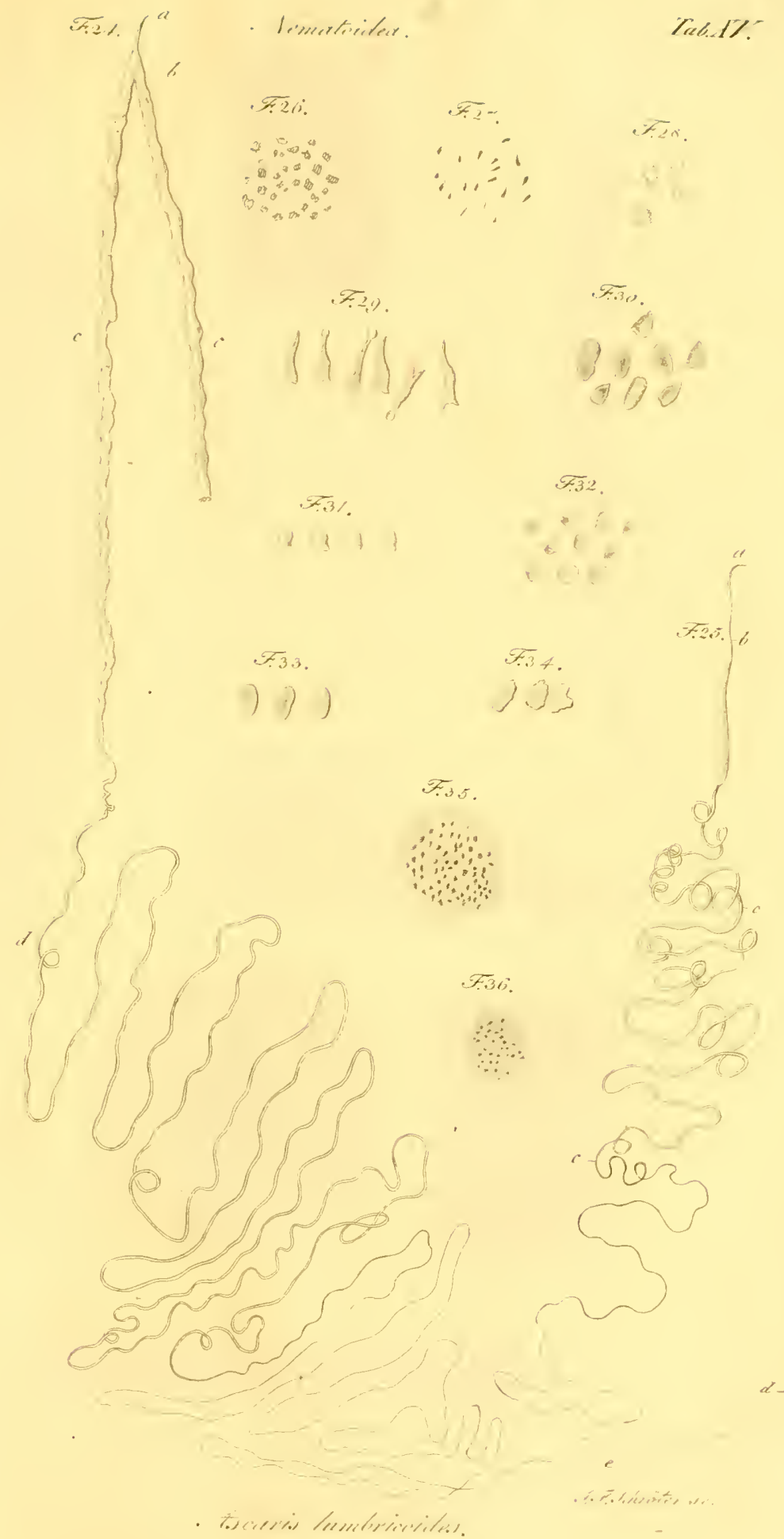





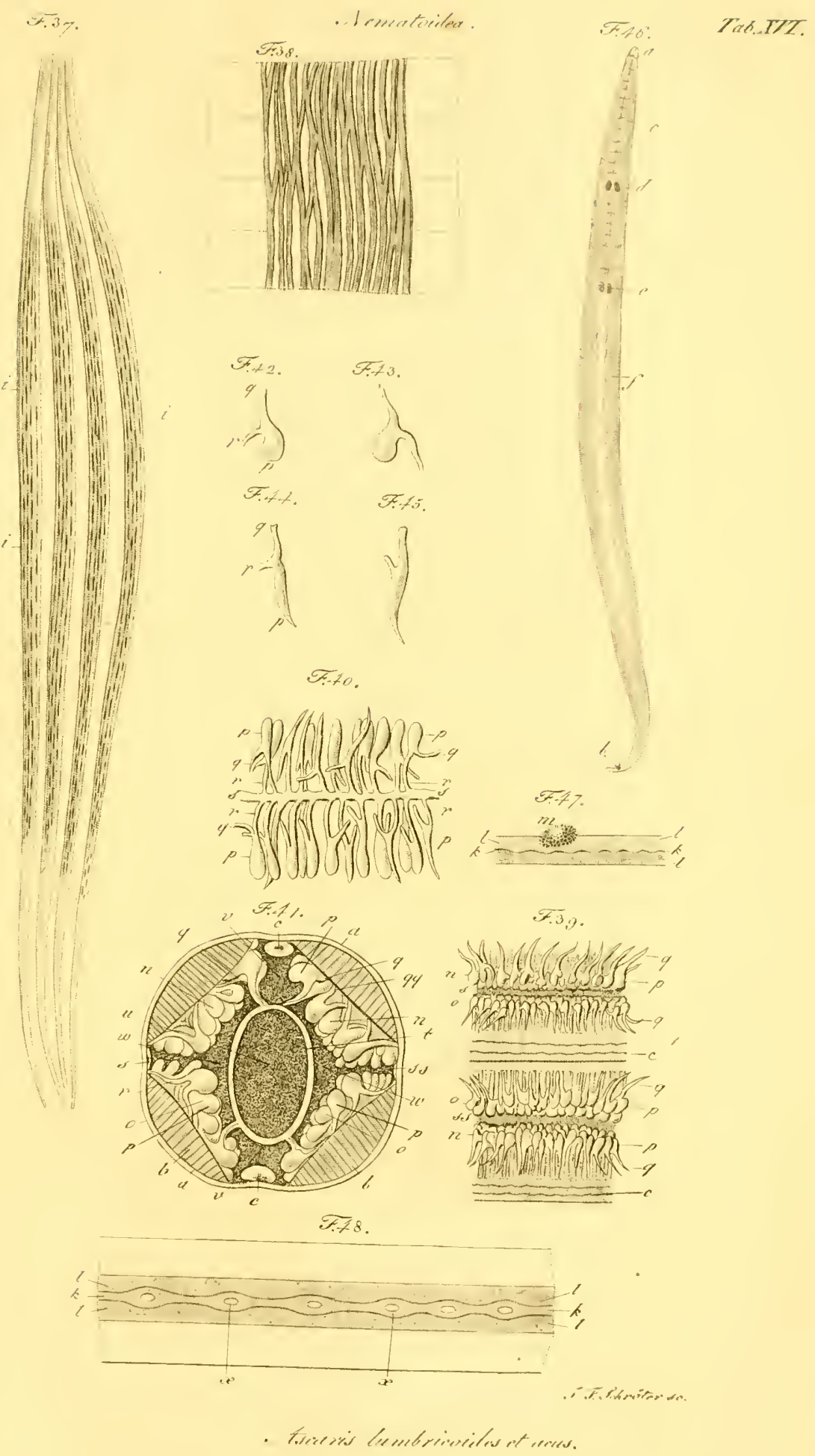





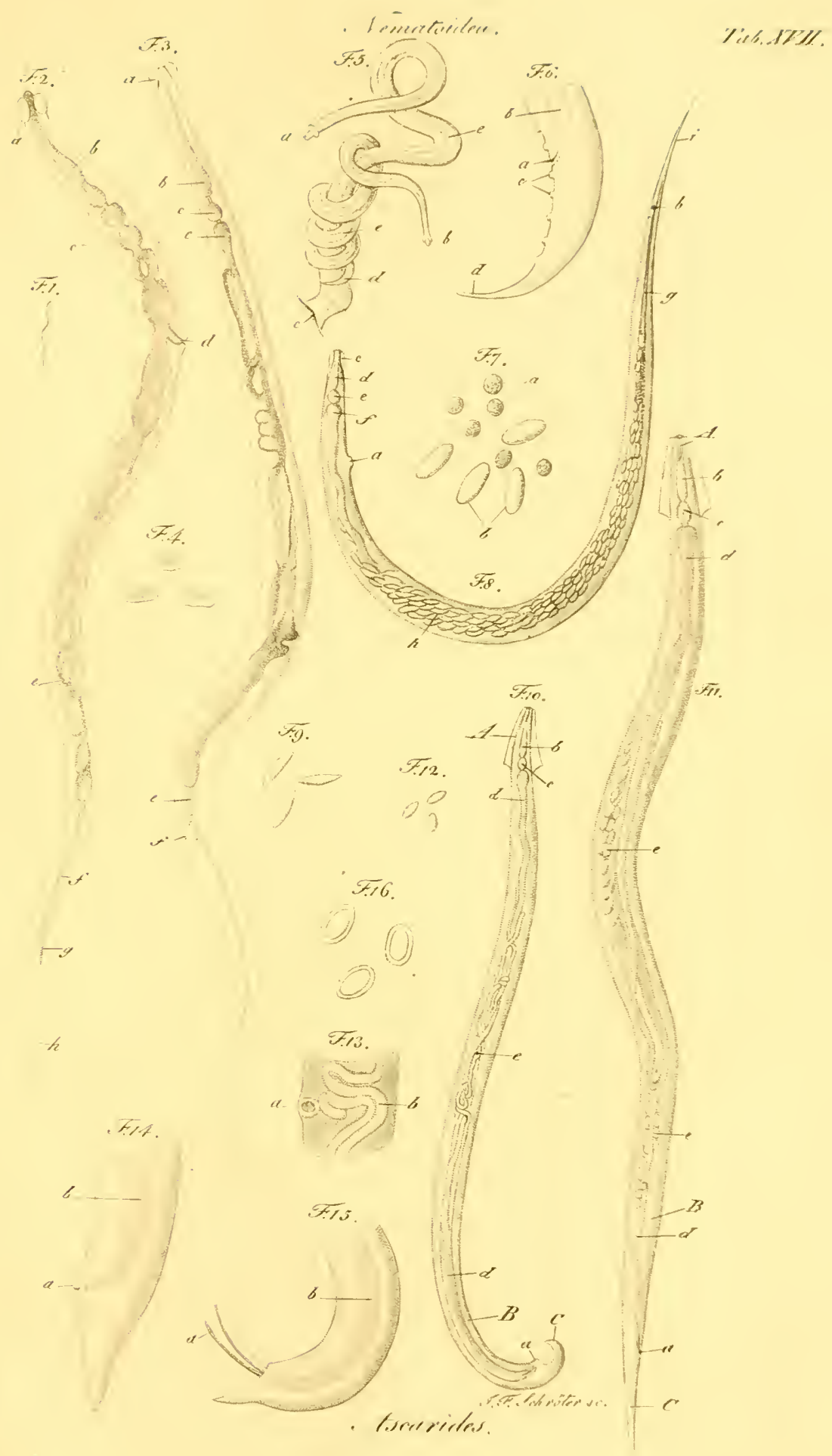




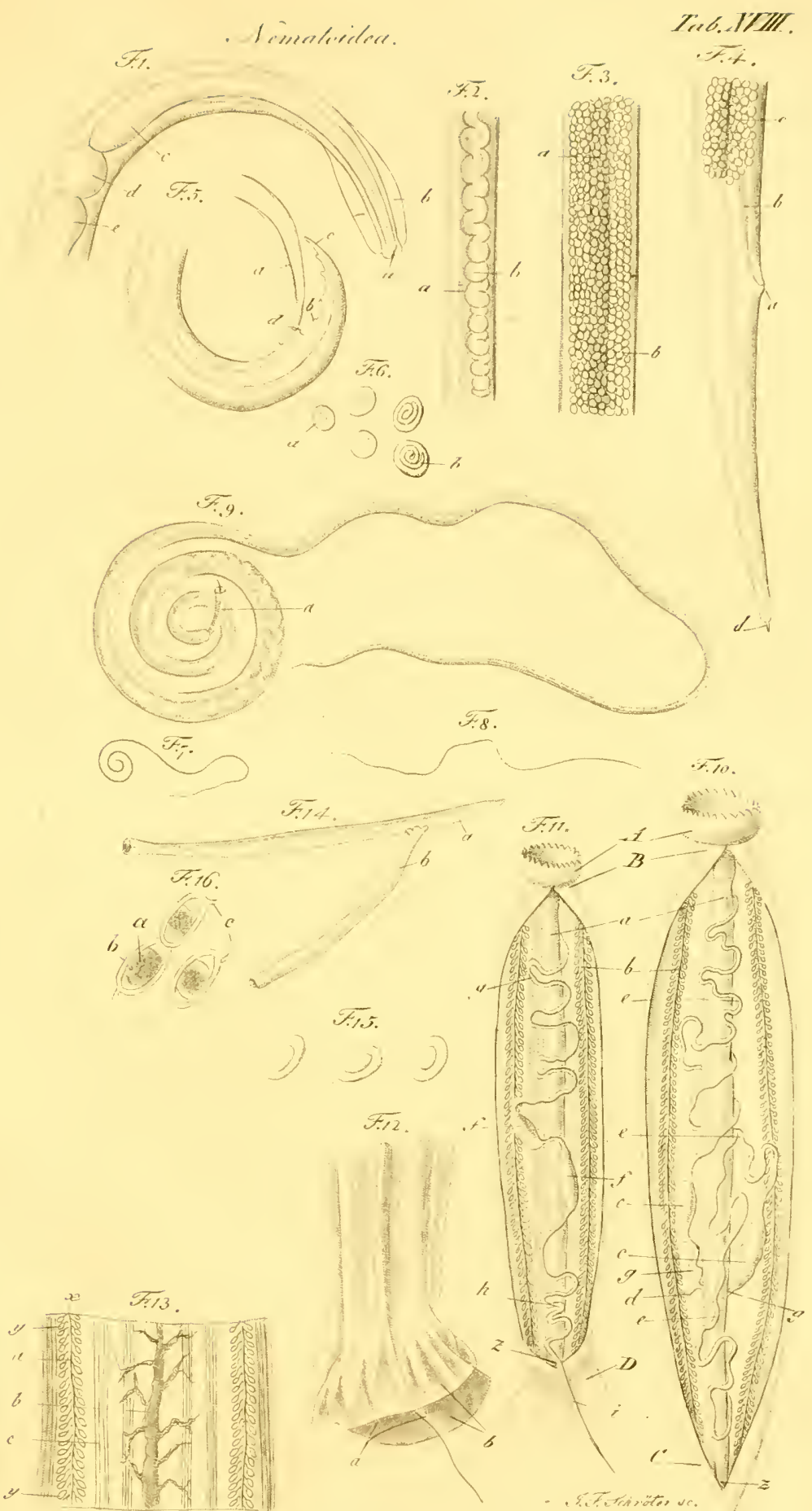

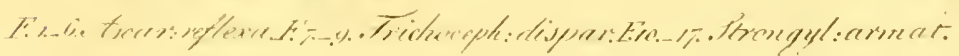





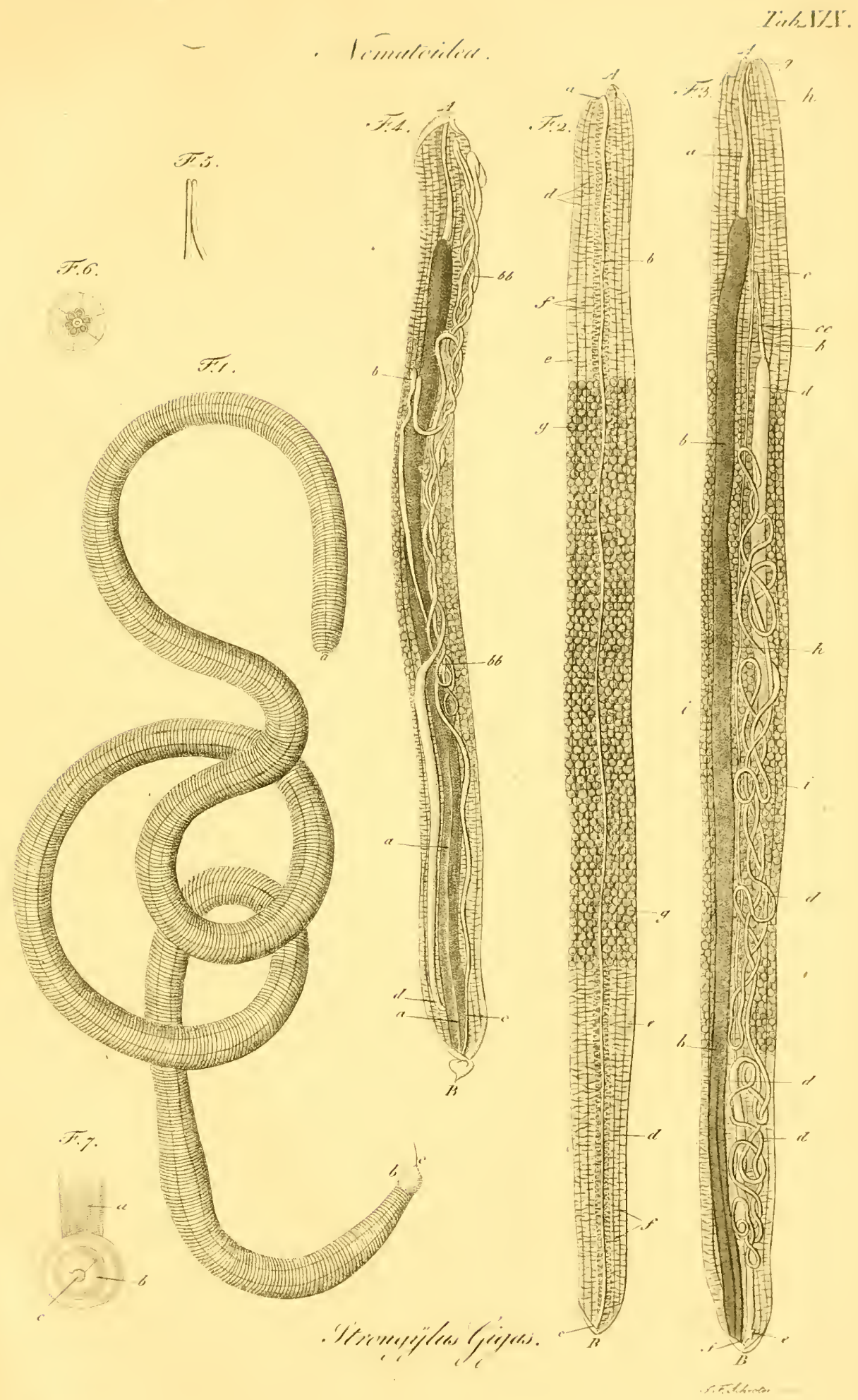







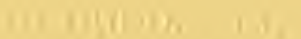

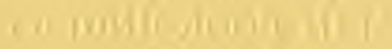

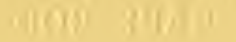





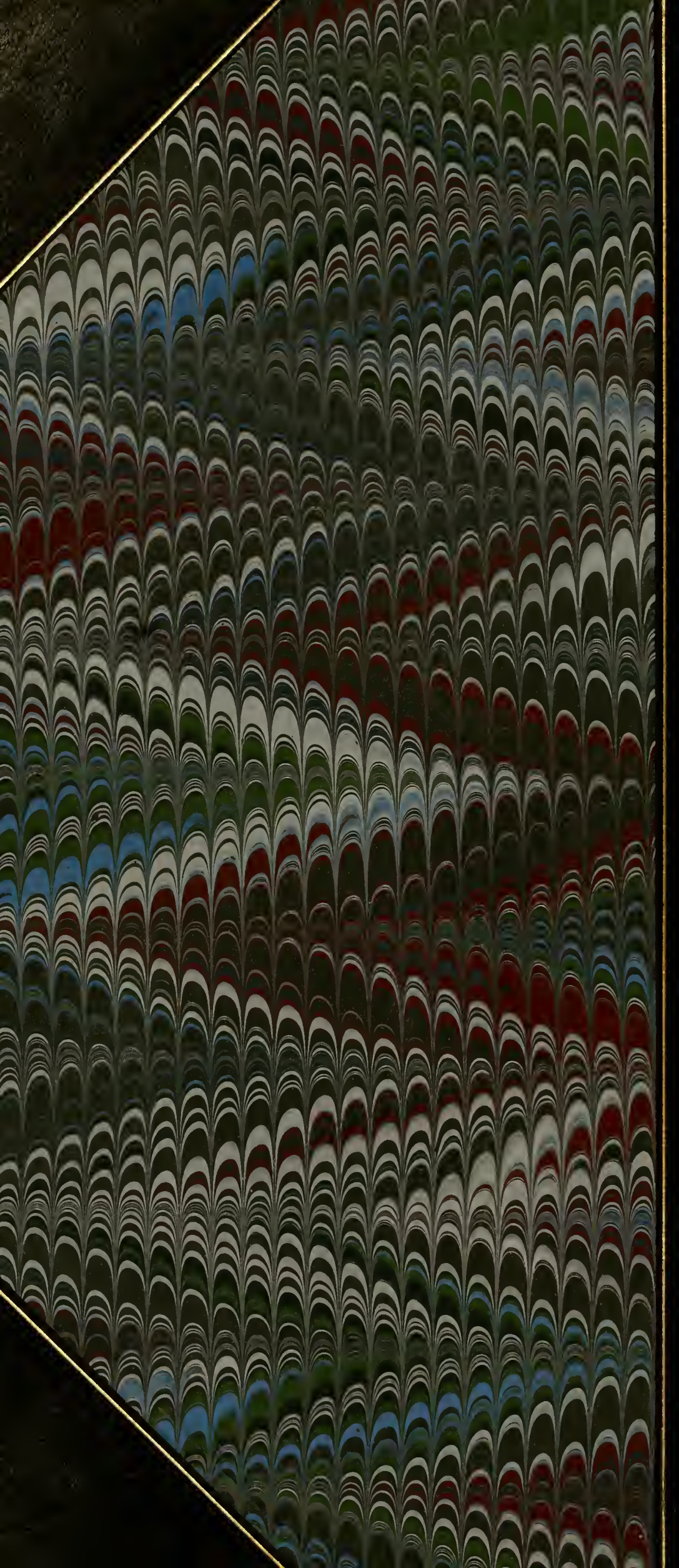

